\title{
Kernos
}

Revue internationale et pluridisciplinaire de religion grecque antique

$23 \mid 2010$

Varia

\section{Ninfe ad Heraklea Lucana?}

Il santuario extra-urbano di Masseria Petrulla nella Valle del Sinni (Policoro - MT)

Ilaria Battiloro, Antonio Bruscella e Massimo Osanna

\section{(2) OpenEdition}

\section{Journals}

Edizione digitale

URL: http://journals.openedition.org/kernos/1584

DOI: 10.4000/kernos. 1584

ISSN: 2034-7871

\section{Editore}

Centre international d'étude de la religion grecque antique

\section{Edizione cartacea}

Data di pubblicazione: 1 gennaio 2010

Paginazione: 239-270

ISSN: 0776-3824

\section{Notizia bibliografica digitale}

Ilaria Battiloro, Antonio Bruscella et Massimo Osanna, « Ninfe ad Heraklea Lucana? », Kernos [En ligne], 23 | 2010, mis en ligne le 10 octobre 2013, consulté le 19 avril 2019. URL : http:// journals.openedition.org/kernos/1584; DOI : 10.4000/kernos.1584 


\title{
Ninfe ad Heraklea Lucana? \\ Il santuario extra-urbano di Masseria Petrulla nella Valle del Sinni (Policoro - MT)
}

\begin{abstract}
During the 1970s, Dinu Adamesteanu uncovered a small sacred place within the chora of Heraklea. It is an open-air sanctuary, constituted by an area bounded by a temenos wall, with an altar and a small naiskos inside. A votive deposit was located within the temenos, which was filled with a large quantity of ritual and votive material, placed in the hole when the sacred place was abandoned. The architectural structures and a selection of the finds were first published by Dinu Adamesteanu himself. He identified this small sacred place with Chalcas' beroon, which is recorded in Lycophron's famous passage.

The subject of this contribution is a comprehensive re-examination of this sacred place - architectural structures as well as published and un-published finds - which allows us to present a different scenario from that first proposed by Adamesteanu.

Most of the Black-Glaze vases pertain to the practice of the symposium, while the common wares suggest that food was usually prepared in order to be given as a gift to deities. The Red-Figure vases present interesting figural depictions, which tend to refer to Dionysian subjects and to the theme of weddings.

With regard to coroplastic objects, three fragmentary life-size terracotta statues have been found. At least two of these represent young, beautiful women, seated on thrones and wearing lavish clothes. A number of small terracotta figurines show diverse iconographies, which pertain to the spheres of kourotropheia and sanatio, but there are also "erotic" subjects, referring to the Aphrodisian sphere, and votive clay fruits, which suggest the chthonian aspect of this cult.

In light of this comprehensive analysis of archaeological data, a clearer and more detailed picture emerges of the Contrada Petrulla sanctuary. The sacred area was probably dedicated to feminine deities, who can be identified with the Nymphs, peripheral figures of Aphrodite's circle. These feminine deities who have chthonian characteristics, personify water (generally a water spring), oversee the passage from a virginal condition to the status of nymphai, have a kourotrophic nature, and are ultimately associated with the Dionysian world. Therefore, the life-size seated statues found in the votive deposit can be considered as cult statues (depictions of the Nymphs themselves?). Previously, the statues had been located in the small naiskos, which was placed within the temenos wall and was open to the altar.
\end{abstract}




\section{Introduzione}

A circa quattro chilometri dalla costa ionica, nei pressi di un piccolo pianoro affacciato sull'antico letto del fiume Sinni e delimitato a nord e a est dal Fosso Petrulla, Dinu Adamesteanu portò alla luce, tra il 1976 e il 1977, un piccolo luogo sacro pertinente alla chora di Heraklea ${ }^{1}$ (Fig. 1).

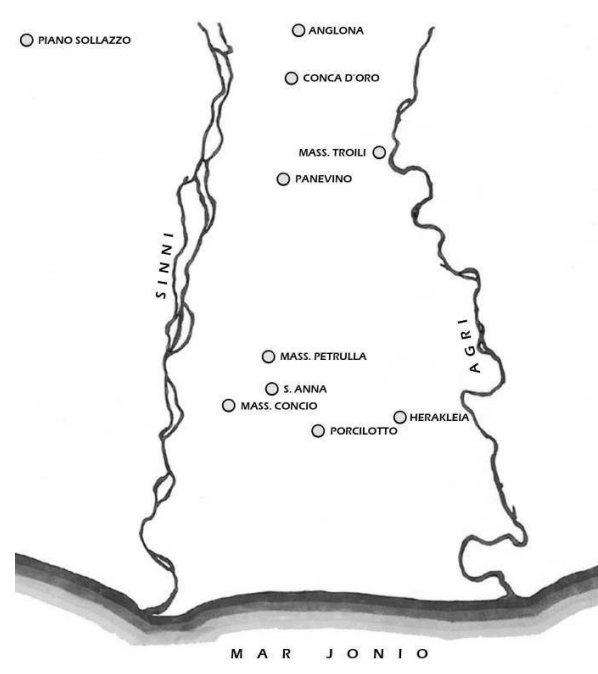

Fig. 1

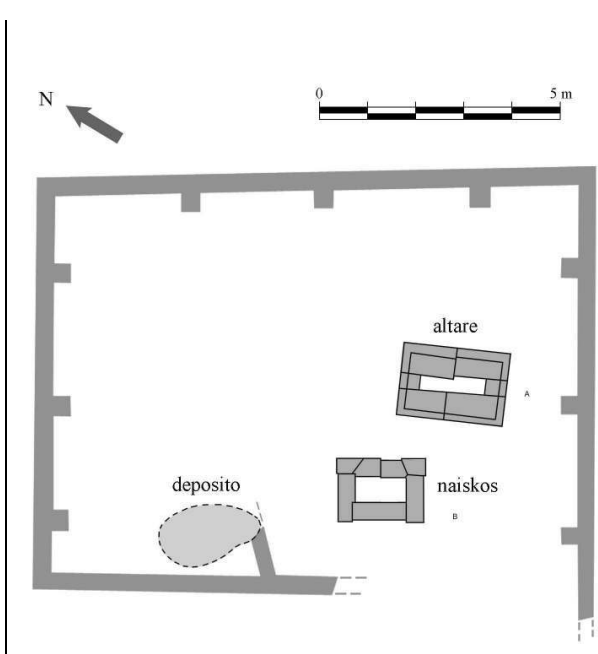

Fig. 2

Posta nei pressi di una sorgente, l'area sacra era delimitata da un muro di temenos, che definiva uno spazio a cielo aperto di forma rettangolare ampio circa $\mathrm{m}^{2} 100$. Al recinto, realizzato con zoccolo in ciottoli e alzato in mattoni crudi, si addossava all'interno, su tre lati, una tettoia o un portico (come attestava al momento dello scavo la distribuzione delle tegole di copertura), mentre l'area centrale risultava occupata da due piccole strutture in blocchi, interpretate rispettivamente come un altare (A) e una edicola (B). Nel settore sud-occidentale, a ridosso del muro sud dell'edificio, lo scavo portò inoltre alla luce un'ampia fossa oblunga e poco profonda, il cui riempimento restituì numeroso materiale votivo e rituale (statuette in terracotta, ceramica, frutta miniaturistica) (Fig. 2).

Per quanto riguarda la cronologia dell'impianto, in base alla sola considerazione della suppellettile rinvenuta all'interno della fossa, è possibile stabilire

${ }^{1}$ In quest'area già il Quilici aveva notato la presenza diffusa di frammenti ceramici, i quali si intensificavano soprattutto sull'arco del ciglio che segue il Fosso. Nel settore più basso rispetto alle terrazze che si elevano lievemente a dominare il fiume, proprio nei pressi del vecchio letto fluviale, in proprietà Cafaro, Dinu Adamesteanu, in seguito ad un sopralluogo effettuato per la segnalazione di materiale fittile e lapideo emerso a causa di profonde arature, intraprese lo scavo che portò alla luce il piccolo luogo sacro: Quilici (1967), p. 148, n. 85; ADAMESTEANU (1977), p. 376-377; ADAMESTEANU (1982), p. 459 sq.; OSANNA et al. (2008), p. 51-56. 
un arco di frequentazione compreso tra il secondo quarto del IV sec. a.C. e il primo quarto del secolo successivo. Per quanto riguarda la fine della frequentazione cultuale, da inquadrare non oltre il primo venticinquennio del III sec. a.C., poco si può arguire dal dato archeologico, che non sembrava documentare una distruzione traumatica. L'interrompersi della frequentazione in un momento compreso entro la prima metà del III sec. a.C., trova comunque un significativo parallelo nella contrazione delle presenze nelle chorai della costa ionica, dove si assiste - a Metaponto meglio che ad Heraklea, grazie alle ricerche più sistematiche ed intensive - al progressivo decremento del numero di fattorie e dunque ad uno sfruttamento meno intensivo della terra ${ }^{2}$. Il rarefarsi delle presenze umane nel territorio deve aver portato con sé all'abbandono di un luogo sacro, evidentemente strettamente connesso allo sfruttamento della chora, e a chi nel territorio viveva.

Interessante notare, comunque, che il grande deposito, il quale raccoglie materiale disomogeneo, tanto dal punto di vista cronologico che tipologico, sembra sia stato realizzato in un unico momento, verosimilmente proprio in relazione all'abbandono del sito, come mostra anche il rapporto stratigrafico tra fossa e strutture del portico, il cui muro obliquo desinente dalla parete sud del temenos risulta parzialmente distrutto proprio dalla realizzazione del deposito stesso. Tale circostanza sembra far pensare che la fossa, in cui era destinato a confluire tutto il materiale votivo e liturgico presente nell'area sacra, sia stata scavata in un momento in cui il portico doveva essere già almeno parzialmente distrutto, altrimenti mal si spiegherebbe che si venga a scegliere per la creazione del deposito una parte del portico, da distruggere all'uopo per realizzare la fossa, mentre all'interno dell'area ipetrale c'era molto spazio a disposizione. [M.O.]

\section{L'area sacra di località Petrulla: le strutture, i materiali}

\section{L'architettura}

Passando a considerare più da vicino gli aspetti architettonici del piccolo complesso, si tratta, come anticipato, di un recinto grosso modo rettangolare (m 11,50 × 8,50 ca.), orientato nord-est/sud-ovest, costituito da un muro, che presentava lungo il suo perimetro una serie di speroni, tre per ogni lato, funzionali alla realizzazione della copertura di un portico con tetto a quattro falde, con gli spioventi inclinati verso l'interno ${ }^{3}$. Non è escluso, del resto, che lo spazio fra uno sperone e l'altro fosse utilizzato per l'inserimento di scaffalature o tavole lignee, funzionali all'esposizione di ex-voto o alla deposizione di offerte,

\footnotetext{
2 OSANNA (1992); CARTER (1987).

3 In generale su "muri tronchi", con o senza colonne all'estremità, sporgenti dalle pareti all'interno di templi o sale per cerimonie religiose in Grecia, cf. DinSMOOR (1950), p. 47, 55, 156, 233, 270. Cf. a Cirene il cosiddetto Portico B4: STuCCHI (1975), p. 64, fig. 50.
} 
secondo un costume diffuso nei santuari sia greci che italicił. È probabile che l'ingresso non conservato si aprisse presso il lato ovest del recinto, in connessione con una lacuna piuttosto ampia.

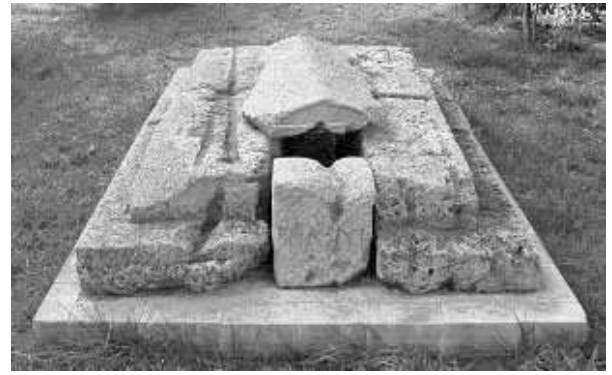

Fig. 3

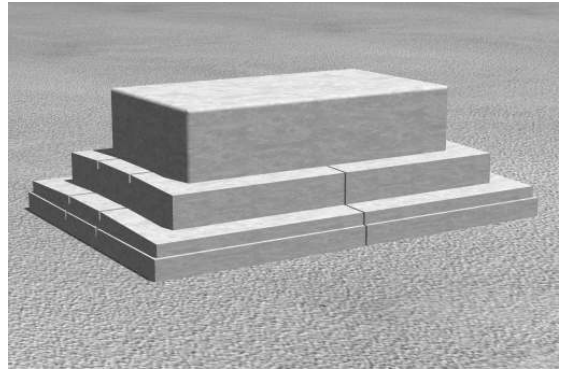

Fig. 4

All'interno dello spazio ipetrale, nel settore sud-orientale - e dunque in stretta relazione con lo spazio in cui è ipotizzabile l'accesso - sono state messe in luce due strutture rettangolari in blocchi di carparo. La prima (A) (m 2,20 × 1,55) (Fig. $3-4)$, realizzata con sei blocchi squadrati dalle dimensioni variabili, può essere agevolmente identificata con un altare, a giudicare dai blocchi che risultano tutti lavorati in maniera analoga, in modo da realizzare una sorta di gradino lungo tutti e quattro i lati. La seconda (B) (Fig. 5-6), posta a m 2 di distanza dalla precedente

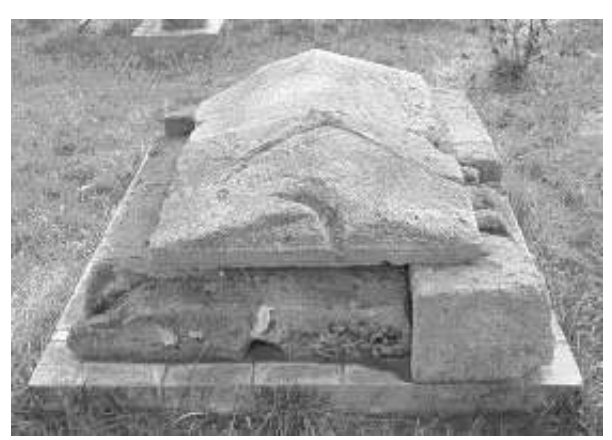

Fig. 5

$(\mathrm{m} 1,90 \times 1,25)$, risulta di più incerta interpretazione: è costituita da cinque blocchi di dimensioni variabili, dei quali quelli posti sul lato est risultano leggermente sporgenti. Il rinvenimento, a non molta distanza dalla struttura, di due blocchi in carparo uguali, lavorati a doppio spiovente con spazio triangolare dei due lati brevi definito da una modanatura aggettante, potrebbe portare, in via ipotetica, ad una ricostruzione del manufatto architettonico come un piccolo naiskos, il cui alzato poteva essere in mattoni crudi $\mathrm{o}$, più verosimilmente, in piccoli blocchetti di carparo ${ }^{5}$.

Se tale ricostruzione coglie nel segno, potremmo essere di fronte ad una associazione estremamente diffusa nel mondo antico, dove una edicola si viene ad affiancare ad un altare, qualificando in questo modo, molto semplicemente,

4 Osanna (2001), p. 113, con bibliografia. Da ultimo, cf. LA TORRE (2002), p. 325-326. In generale sull'offerta di cibo alla divinità, $c f$. BOUMA (1996), p. 109.

${ }^{5}$ Questo schema architettonico è diffuso nel periodo ellenistico: WEBER (1990), p. 58. 
lo spazio sacro ${ }^{6}$. Per restare in ambito geografico prossimo a quello in questione, si può citare il caso del santuario urbano di Dioniso, dove, nelle adiacenze dell'altare, è stato scavato per l'appunto un piccolo naiskos (associato ad un piede di statua marmorea, appartenente forse a quella di culto ospitata originariamente al suo interno $)^{7}$.

Esprimersi sulle caratteristiche formali di questa struttura risulta alquanto difficile, anche perché manca, ad oggi, uno studio tipologico complessivo su questa categoria di monumenti ${ }^{8}$. Di fatto, la maggior parte della documentazione proviene soprattutto dalle raffigurazioni della ceramica funeraria apula. Rispetto all'aspetto canonico che i naiskoi presentano sulla ceramica, nel nostro caso è verosimile che il monumento fosse aperto sul lato lungo, presentando la fronte rivolta verso l'altare ${ }^{9}$ (Fig. 6).

Una soluzione di questo tipo, sia pur meno consueta di quella comunemente attestata, potrebbe
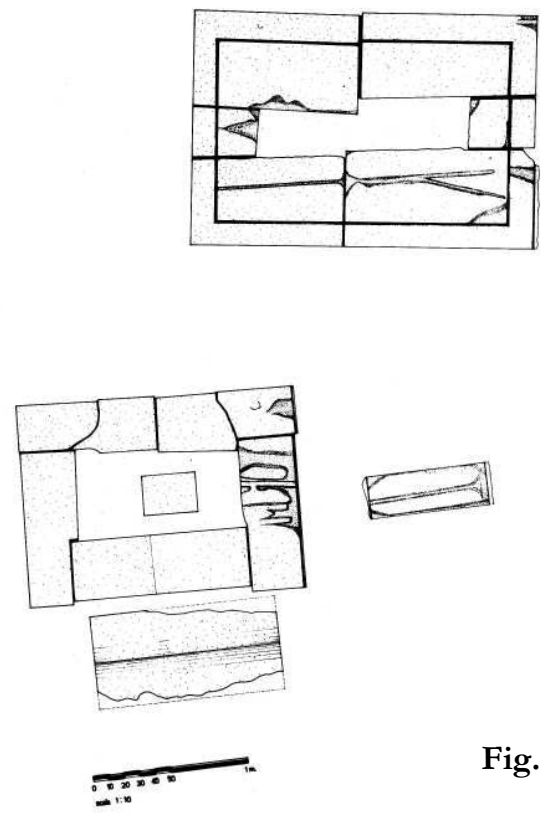

Fig. 6 essere considerata verosimile, visti alcuni confronti tratti dalle riproduzioni fittili, in piccolo formato, di monumenti "reali"10. Tra l'altro tale soluzione, in riferimento all'area sacra di località Petrulla, avrebbe meglio risposto ad una logica rituale, dove la statua o le statue di culto solitamente sono rivolte verso l'altare.

\footnotetext{
6 WEBER (1990), p. 71.

7 PIANU (1996), p. 71-72, tav. 4. In ambito italico, e in riferimento ad un santuario non urbano, possiamo citare il caso dell'area sacra di Fontana dei Monaci a Banzi: MASSERIA (1999), p. 471.

8 Lippolis (1994), p. 112. Interessanti spunti offre un contributo dello stesso autore in cui vengono affrontati diversi problemi inerenti l'architettura funeraria dell'Apulia ellenistica, nel quale ampio spazio viene lasciato appunto a questa categoria di monumenti: cf. LIPPOLIS (1987), p. 146 sq., fig. 32-36, tav. 15-17.

${ }^{9}$ Weber (1990), p. 66-67; Trendall (1953), p. 208-209, tav. LV, d. AA. 4; PONTRANDOLFO et al. (1988), fig. 43, n. 1-2; fig. 44, n. 1-5.

10 Cf. Staccioli (1968), p. 50-51, tav. LII-LIII, 1, n. 41; 63-64, tav. LXIII-LXIV, n. 57; su quest'ultimo esemplare ved. JOSEP (1997), p. 229-230 (fig. a p. 230), con bibliografia precedente.
} 


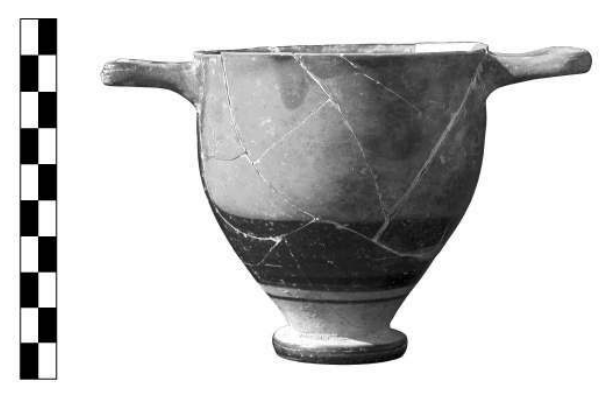

Fig. 7

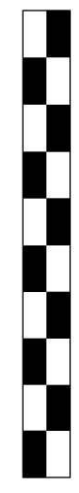

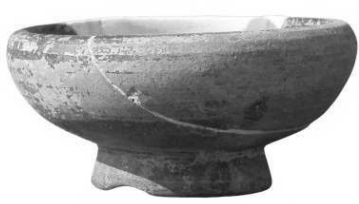

Fig. 9
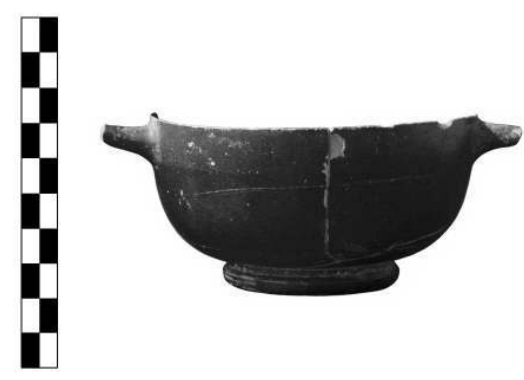

Fig. 8

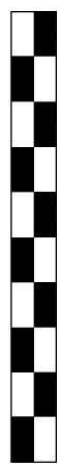

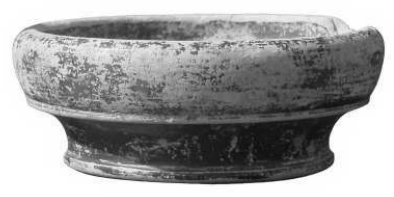

Fig. 10

\section{Il deposito votivo: la ceramica}

Come si è anticipato, il materiale votivo proviene per la maggior parte dalla fossa ubicata nel settore sud-occidentale del recinto (m 2 x 1,30) (Fig. 2).

La ceramica a vernice nera, inquadrabile entro un arco cronologico compreso tra la metà del IV e gli inizi del III sec. a.C., è rappresentata principalmente da forme che rientrano nei repertori tipici dei contesti della Lucania meridionale e dell'Apulia centro-meridionale. Prevalgono senza dubbio le forme aperte, il cui repertorio comprende soprattutto skyphoi di tipo sia attico che corinzio (Fig. 7) e coppe biansate su basso piede ad anello modanato (Fig. 8). Quasi insignificante il numero degli esemplari riconducibili ad altre forme, generalmente rappresentate da pochi o addirittura un solo esemplare: per esempio, l'unica coppetta ad orlo rientrante (Fig. 9) o quella a profilo concavo-convesso (Fig. 10). Di relativo pregio risulta una kylix (Fig. 11), purtroppo frammentaria, caratterizzata da un motivo 
vegetale ottenuto a stampo impresso al centro della vasca ${ }^{11}$. Pochissimi sono, invece, i frammenti riferibili a forme chiuse ${ }^{12}$, in particolar modo a lekythoi baccellate e a corpo liscio.

Per quanto concerne la ceramica a figure rosse, la maggioranza degli esemplari appartiene a forme aperte, in particolare vasi per bere. Particolarmente numerosi i vasi di grandi dimensioni come crateri, hydriai e skyphoi (Fig. 12). Inoltre, si segnala il rinvenimento di un certo numero di frammenti relativi a glaukes. Non mancano, pur se rappresentati da frammenti riconducibili ad un unico individuo, forme quali oinochoe, pelike, anfora.

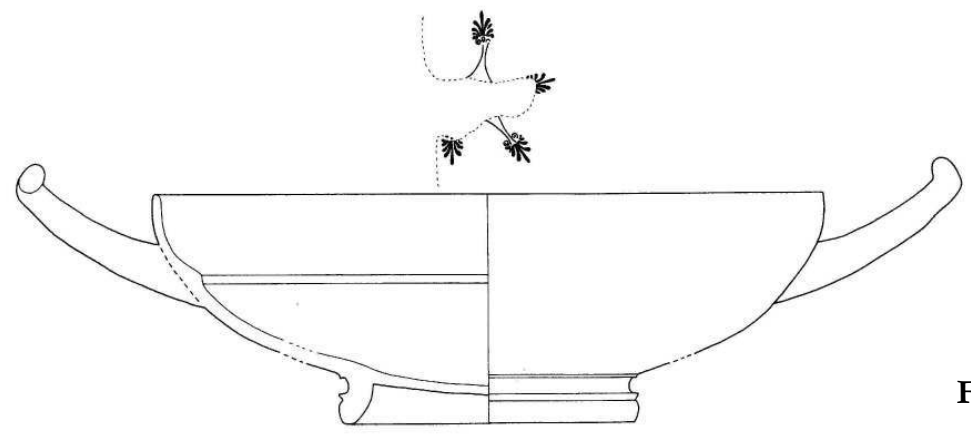

Fig. 11

Le immagini, laddove ricostruibili, rimandano insistentemente al mondo di paides e parthenoi, o a scene dionisiache (menadi o ninfe riprese in una danza estatica nell'atto di percuotere un timpano, associate a satiri o ad auleti che ne scandiscono il ritmo ${ }^{13}$ ), ma si segnalano anche alcune interessanti scene riferibili al mito (Fig. 13-20).

Tra le scene legate al mito, si segnala un frammento di cratere con la raffigurazione del mito di Bellerofonte e la Chimera. La presenza di una corona all'altezza della testa di Bellerofonte rievoca il momento della incoronazione dell'eroe, piuttosto che la fase precedente del combattimento con il mostro ${ }^{14}$ (Fig. 21).

11 Rispetto ai modelli greci, la produzione magnogreca si diversifica per un'evidente semplificazione dei motivi a stampo: SPARKES, TALCOTT (1970), p. 102-105, tav. 22-23, fig. 483, 484, 487, 496.

12 Questa circostanza, molto probabilmente, è il risultato di una selezione effettuata in base ad esigenze rituali e sacre. Lo stesso dato quantitativo emerge per esempio ad Armento, nel santuario in località Serra Lustrante: Russo TAGLIENTE (2000), p. 80.

${ }^{13}$ Su questa sfera iconografica, legata alla musica, si rimanda a RESTANI (1991), p. 379 sq. La danza estatica è ritenuta una componente fondamentale degli aspetti legati alla sfera dionisiaca: JEANMAIRE (1949), p. 463-473. Sul ruolo fondamentale di queste figure nell'ambito del corteggio dionisiaco $c f$. inoltre NiLSSON (1952), p. 113.

14 Sulla sezione del mito relativa alla lotta di Bellerofonte contro la Chimera ved. KERÈNYI (1984), p. 89-90. Da ultimo, sulla ricezione di questo mito in Occidente, sia in ambito greco che indigeno, ved. Mugione (2000), p. 96-97; POUZADOux (2002), p. 124-125. 


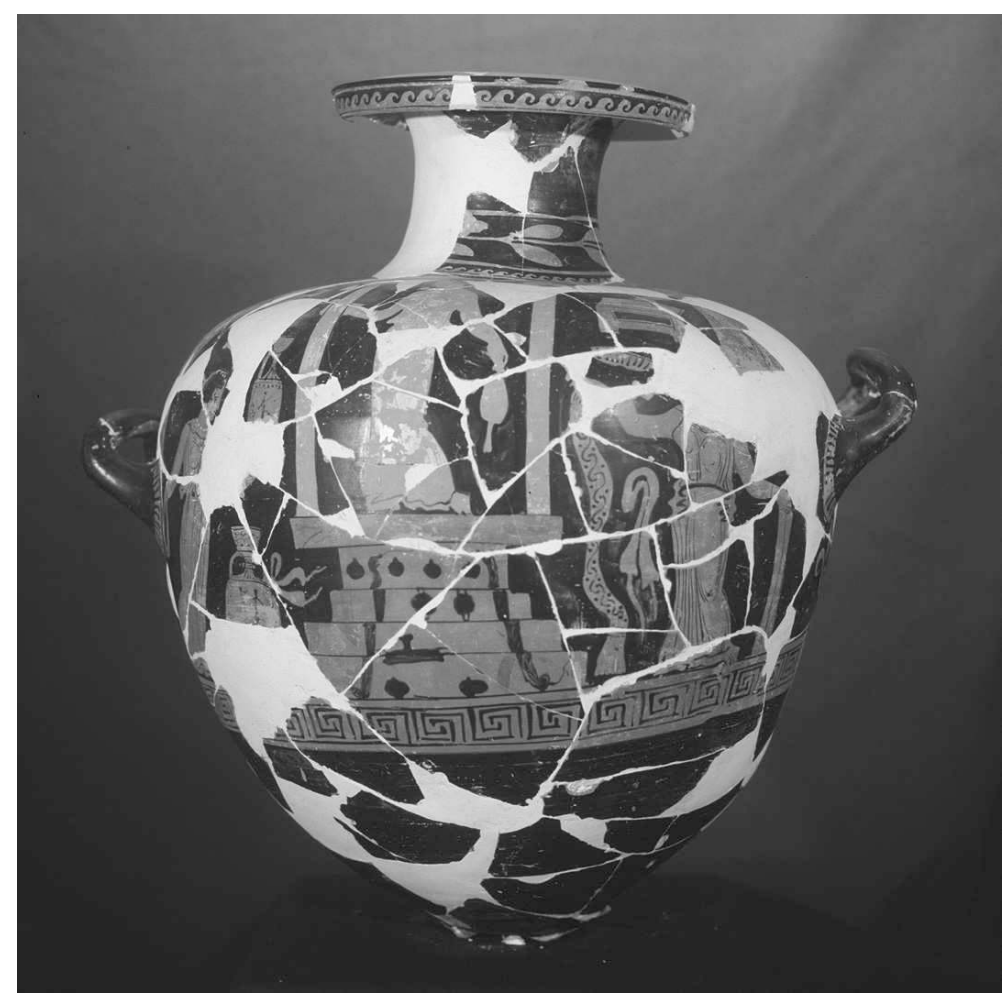

Fig. 12

Su una bydria frammentaria è verosimilmente rappresentata la scena del giudizio di Paride ${ }^{15}$ (Fig. 22), come indicherebbe la presenza di Atena in associazione all'eroe troiano. Ad Atena rimandano, infatti, l'egida e l'elmo recati da una delle due figure femminili16, mentre l'elmo alato e l'abbigliamento frigio, tipicamente "troiani", permettono di qualificare la figura maschile come quella di Paride ${ }^{17}$.

15 Cf. Bini (1989), p. 20, n. 38-40 Cf. A. Kossatz-Deissmann, s.v. "Paridis iudicium", LIMC VII, 1 (1994), p. 113, n. 38. Un ricco repertorio iconografico circa questo tema mitico, con particolare rilievo alla produzione apula, è in MORET (1978), p. 76-98, tav. 21-28. È interessante notare, inoltre, come in questo contributo venga affrontato anche un aspetto ideologico e culturale sotteso da questo tema, quello del persistente antagonismo tra Grecia ed Asia ( $c f$. in part. p. 92 sq.).

16 Sull'iconografia di Athena con questo tipo di copricapo, of. da ultimo Mugione (2002), p. $63-80$.

${ }^{17}$ L'elmo frigio, infatti, costituisce un diretto richiamo a Troia, assumendo quasi una precisa connotazione "etnica": Breglia Pulci Doria (2002), p. 104-105; Cambitoglou, Chamay (1997), p. 241, n. 103. 


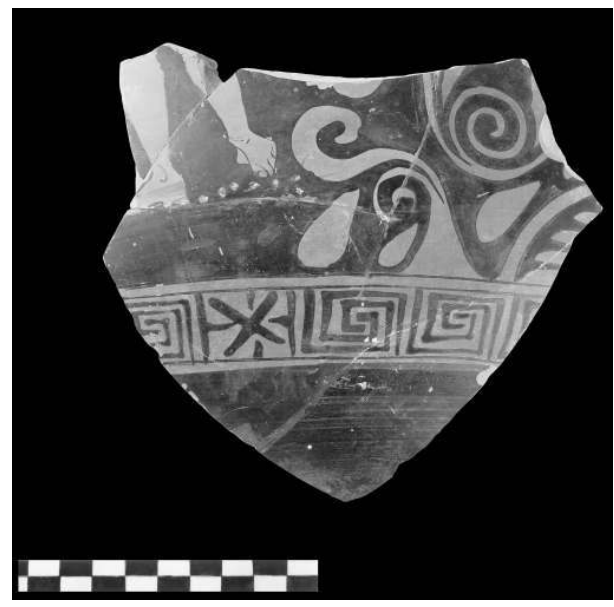

Fig. 13

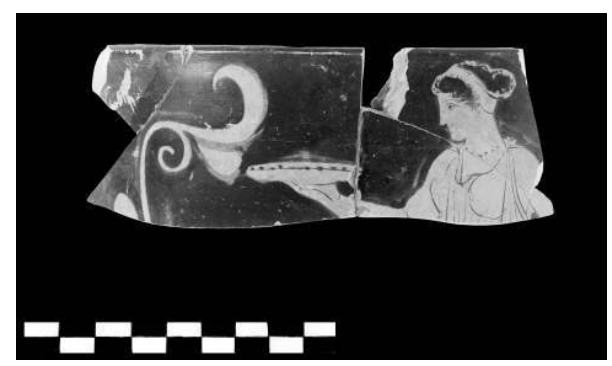

Fig. 15

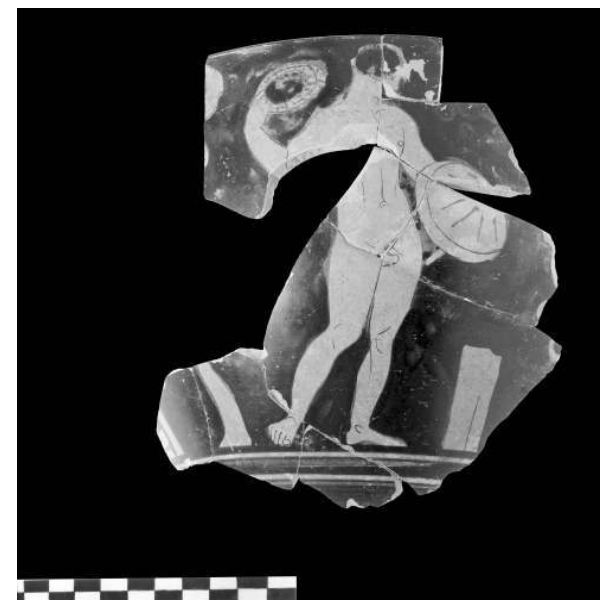

Fig. 14

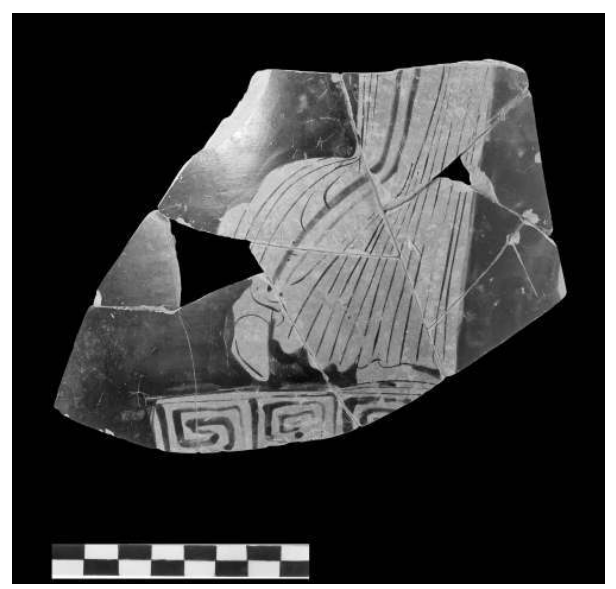

Fig. 17

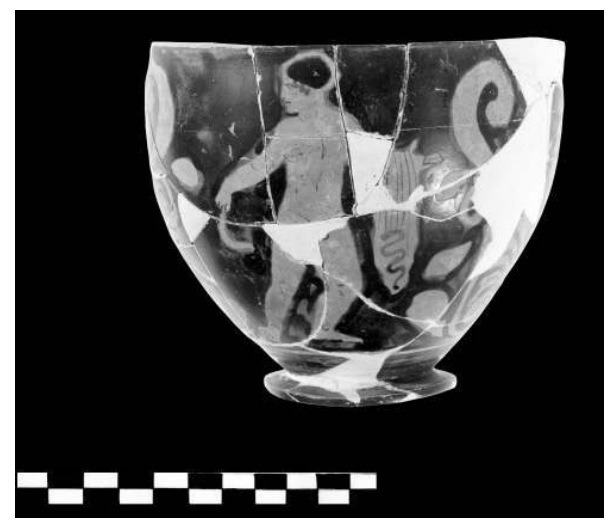

Fig. 16a

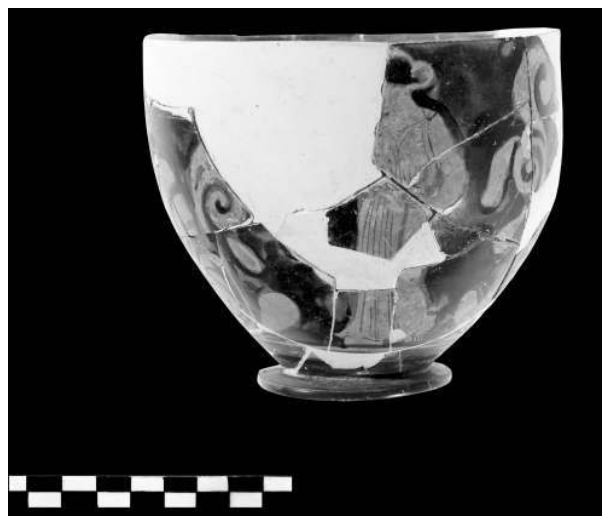

Fig. 16b 


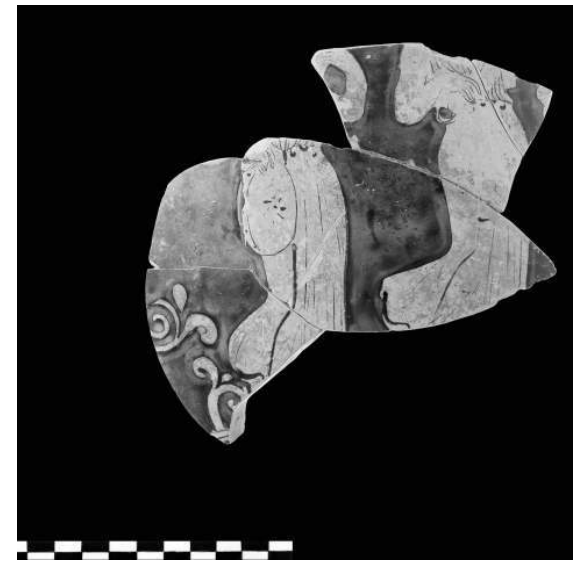

Fig. 18

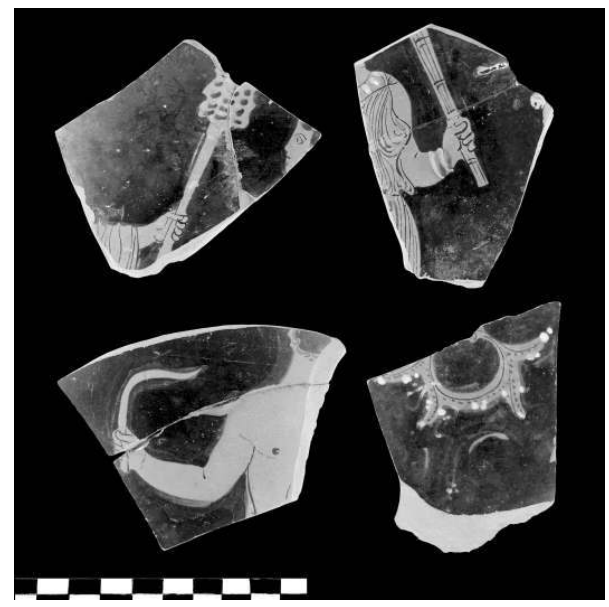

Fig. 20

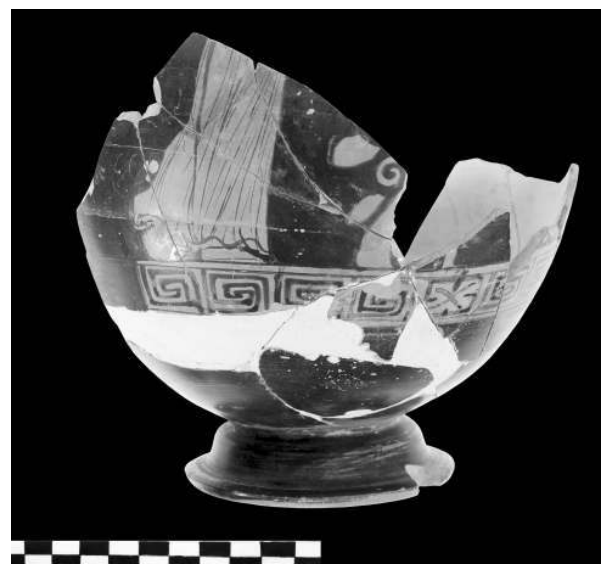

Fig. 19

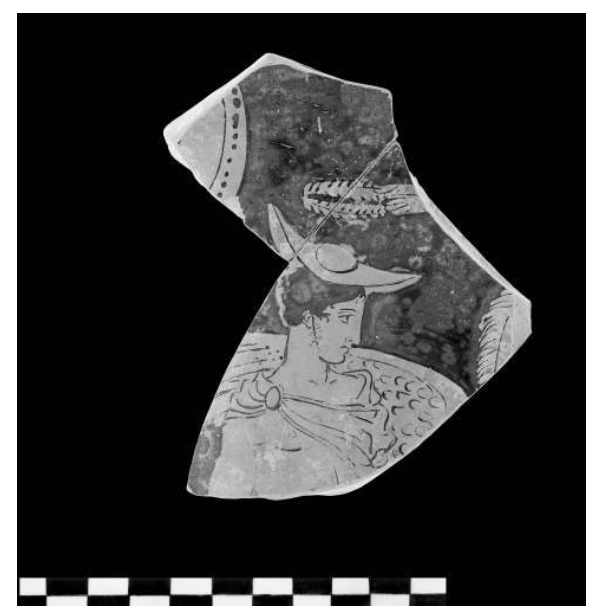

Fig. 21

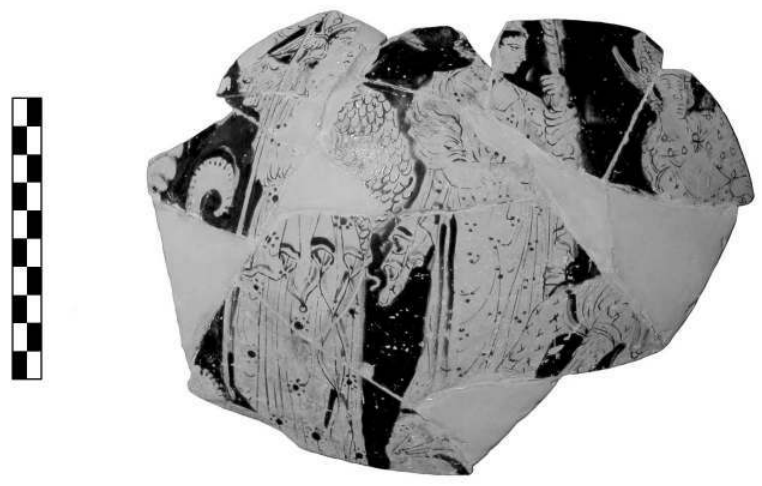

Fig. 22 
Ben più complessa la lettura di un frammento di cratere (Fig. 23), che reca la rappresentazione di più figure che sembrano prendere parte ad una processione marina, come indicherebbe la presenza di un vecchio recante un remo e il fatto che le figure sono tutte rivolte in un unico verso. Si potrebbe pensare alla raffigurazione di un corteo nuziale, forse quello per le nozze di Peleo e Teti, soprattutto in virtù della presenza di una nike in volo, ripresa nell'atto di incoronare, e di un ippocampo, animale marino che ricorre di frequente nella scena delle nozze della divinità marina con l'eroe tessalo, in particolar modo nella ceramica a figure rosse apula ${ }^{18}$.

Fig. 23

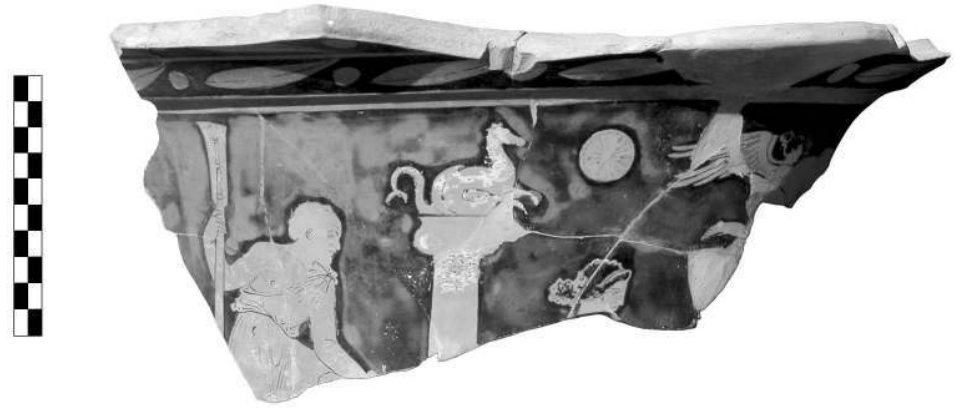

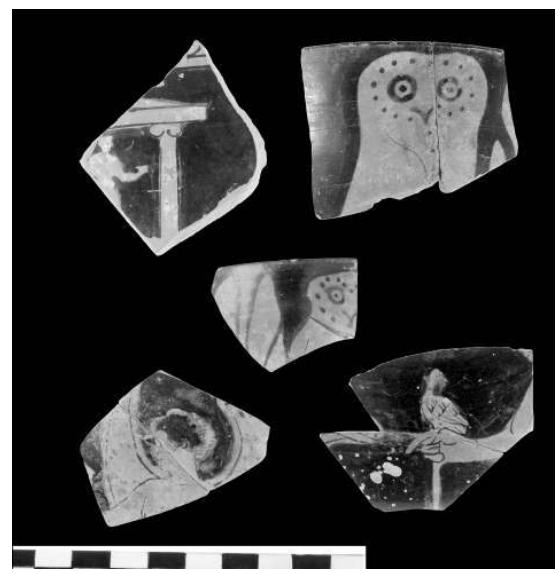

Fig. 24

Altri soggetti iconografici ricorrenti sono la civetta fra rami di alloro ${ }^{19}$, che caratterizza la decorazione degli skyphoi ${ }^{20}$, figure entro naiskoi, immagini alate (Fig. 24).

Si tratta di prodotti assegnabili alla produzione dell'Apulo antico e, in minima parte, al medio, i quali rientrano nella produzione di una ristretta cerchia di pittori: di Sisifo, di Gravina, dell'Ilioupersis, di Licurgo e delle rispettive botteghe, ponendosi cronologicamente nei primi tre quarti del IV sec. a.C. ${ }^{21}$. [A.B.]

\footnotetext{
18 Cf. R. Vollkommer, s.v. "Peleus und Thetis", LIMC VII, 1 (1994), p. 256, n. 60, p. 263,

${ }^{19}$ In generale sugli skyphoi con civetta ved. JOHNSON (1955), p. 119 sq. Sull'utilizzo a scopo rituale di questa particolare categoria di vasi ved. GIORGI et al. (1988), p. 227.

${ }^{20}$ In un solo caso, esso compare anche su una coppa biansata, cogliendosi tuttavia solo a livello delle zampe a causa della lacunosità del frammento.

${ }^{21}$ In generale sull'inquadramento cronologico dello stile proto-apulo, apulo antico, medio e tardo si rimanda, da ultimo, a LipPolis (1996), p. 377-393, con relativa bibliografia; De JULIIS (2004), p. 145-149.
} n. 180. 
Fig. 25

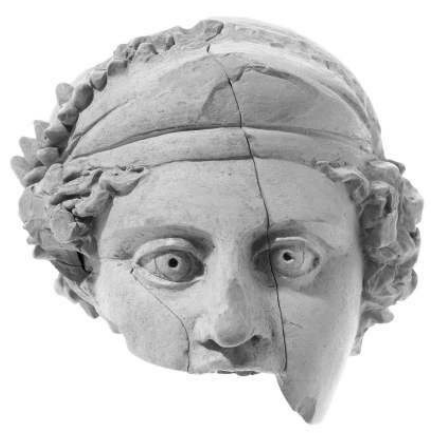

a

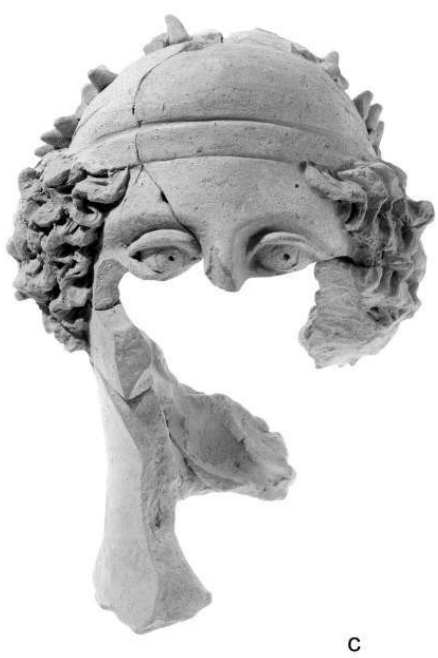

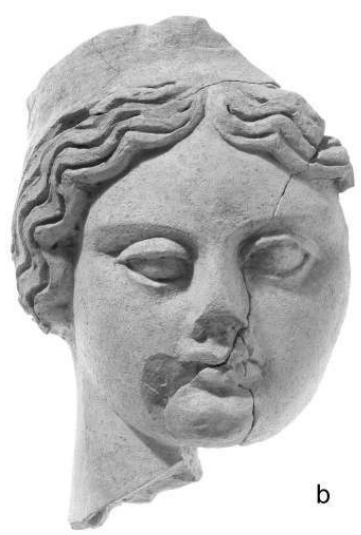

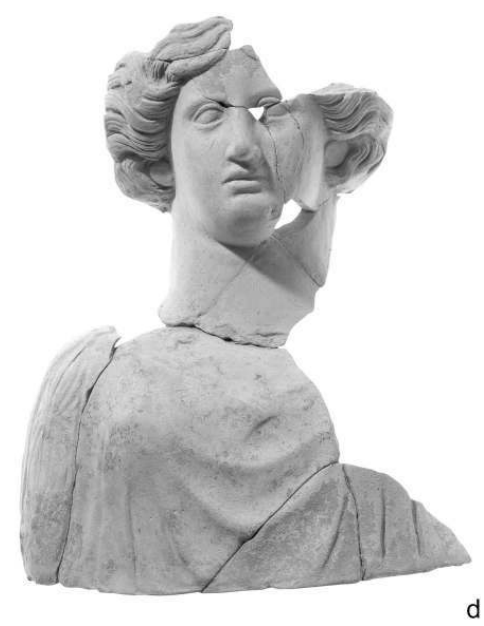

\section{Il deposito votivo: le terrecotte figurate}

La coroplastica rinvenuta nell'area sacra di Contrada Petrulla comprende un numero assai ristretto di esemplari riconducibili sia a plastica di grandi dimensioni che alla categoria delle piccole terrecotte figurate.

La plastica di grandi dimensioni è rappresentata da pochi pezzi frammentari (tre teste femminili e alcuni frammenti pertinenti al corpo di almeno due figure sedute), che si distinguono per fattura particolarmente pregevole. 
Delle tre teste rinvenute, tutte di dimensioni di poco inferiori al vero, una reca sul capo un diadema (Fig. 25b); i capelli rifiniti a stecca e scriminati al centro della fronte a formare due rigide bande, la resa degli occhi con le palpebre rilevate, sono elementi che potrebbero rimandare ai caratteri tipici dello stile "severo" 22 . Tuttavia l'ovale pieno del viso, all'interno del quale spiccano labbra sinuose e carnose, suggeriscono piuttosto una datazione nell'ambito del IV sec. a.C., analogamente a quella delle altre teste.

Le altre due teste (Fig. 25a, c) risultano molto simili, pur non essendo riconducibili alla stessa matrice. Destinate ad una visione frontale, in quanto il retro è grossolanamente lisciato a mano, dovevano verosimilmente appartenere a statue sedute su trono, come attesterebbe la pertinenza di una delle due teste (Fig. 25c) al busto frammentario rinvenuto (Fig. 26), pur in mancanza di punti di attacco. Le figure presentano un'acconciatura con i capelli raccolti in un sakekos o kekryphalos, un copricapo che non rientra specificamente in alcuna iconografia divina $^{23}$ e che costituisce un usuale elemento delle acconciature femminili anche in casa $^{24}$. Sul capo è una corona di elementi conici ${ }^{25}$, probabilmente ghiande ${ }^{26}$. Il modellato del volto, plastico e ben rifinito, con il particolare della resa degli occhi, in cui pupilla e iride sono resi con incisioni, consente una datazione nel corso del IV sec. a.C. Dal punto di vista stilistico, infatti, pur mancando confronti significativi con altri esemplari provenienti dal mondo magno-greco, chiaro è il richiamo di questi pezzi alla tradizione iconografica della statuaria e della coroplastica tarantina di età tardo-classica ${ }^{27}$. Inoltre, poiché la plastica in terracotta di grandi dimensioni non risulta particolarmente diffusa in ambito eracleota, sembra probabile che questi pezzi provengano da atelier di Taranto stessa, con la quale Heraklea, colonia tarantina, era in strettissimi contatti.

Passando a considerare gli altri esemplari di statuaria di grandi dimensioni, si tratta di parte di un busto e un frammento di base di trono. Il primo raffigura il braccio di una figura femminile assisa su trono con braccioli (Fig. 26), vestita di chitone a maniche lunghe abbottonato sulla spalla, le cui pieghe sono rifinite

22 Cf. un esemplare dal modellato affine in LATTANZI et al. (1996), p. 189, fig. 13.32.

23 Che $\mathrm{i}$ due termini siano stati usati in antico come sinonimi è sottolineato in $\mathrm{CH}$. Daremberg, E. SAglio, s.v. "Saccus", Dictionnaire des antiquités grecques et romaine, IV, Graz (196263). Raramente le divinità sono raffigurate con questo tipo di copricapo. Si pensi, ad esempio, al noto caso della "Persefone di Taranto": Belli PasQua (1995), p. 39-43. Numerosi, invece, gli esemplari di figure non divine che recano sul capo il sakkos: LETTA (1971), p. 93, tav. XV,1; 100101, tav. XVII, 3-4; 118, tav. XXIII, 1; IACOBONE (1988), p. 135, tav. 128, a,c,d.

${ }^{24}$ Forti, StAZio (1983), p. 690.

${ }^{25}$ L'associazione del sakkes con una corona di edera ricorre in alcune statuette di piccolo modulo dal Museo Nazionale Jatta di Ruvo (VentrelLi (2004), p. 36-37).

${ }^{26}$ Cf. le corone di ghiande in terracotta provenienti dalla necropoli tarantina di Contrada Vaccarella (Museo (Il) di Taranto (1988), tav. LXXXV n. 34.5c; 426).

${ }^{27} \mathrm{La}$ resa della cavità orbitale delle statue riflette fortemente l'influenza di Taranto, come testimoniato dalla statuaria in pietra tenera tarantina: BERNABÒ BREA (1952), p. 5-241, in part. p. 70; ORLANDINI (1983), p. 428 sq. 
con incisioni a stecca. Un secondo frammento (Fig. 27) è pertinente alla parte inferiore di una figura panneggiata assisa su thronos dai piedi modellati a forma di zampe leonine.

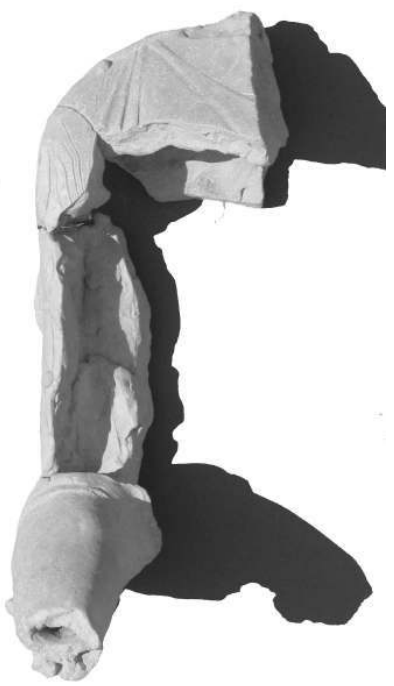

Fig. 26

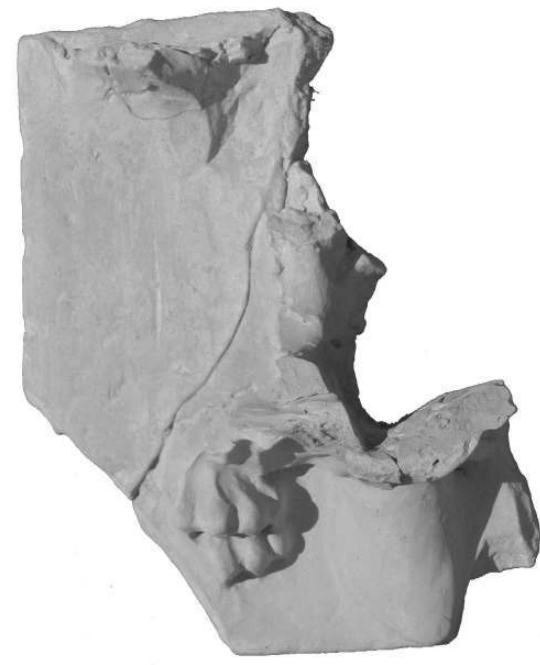

Fig. 27

Prima di passare a considerare la piccola coroplastica, è necessario presentare alcune riflessioni riguardo la funzione e l'identificazione dei soggetti sottesi alle statue appena descritte.

Se è impossibile avanzare ipotesi sulla ricostruzione del tipo cui ricondurre la testa con diadema (Fig. 25b), vale la pena soffermarsi sulle due teste iconograficamente simili, caratterizzate da acconciatura con sakkos e corona di ghiande (Fig. 25a, c). Come si è detto, la testa leggermente più piccola tra le due (Fig. 25c) può appartenere al medesimo esemplare cui va riferito il frammento di busto conservatosi (Fig. 26), che rappresenta una figura femminile assisa. L'unico altro frammento riferibile ad una figura seduta riproduce la parte inferiore di una figura panneggiata, seduta su un trono dai piedi modellati a forma di zampa leonina (Fig. 27). Sulla base di questi dati, sembra potersi ricostruire un tipo di figura femminile panneggiata seduta e riccamente abbigliata, anche se tale lettura - in mancanza di esemplari meglio conservati provenienti da altri contesti - non può considerarsi sicura. Va da sé, inoltre, che - in mancanza di attributi diagnosticamente significativi - l'identità di queste figure è destinata a rimanere sconosciuta.

Nonostante le difficoltà di lettura appena sottolineate, non mancano spunti di riflessione. La rappresentazione di una giovane donna vestita in maniera ricercata, con un copricapo tipico delle donne comuni, poteva essere una rappresentazione volutamente generica della nymphe, la giovane alla vigilia delle nozze. Questo elemento rimanda ad un aspetto iniziatico, che doveva essere di primaria importanza nell'ambito del contesto sacro che si sta analizzando. 
Tuttavia, piuttosto problematica risulterebbe, a questo punto, la presenza del trono, tipico appannaggio di immagini divine ${ }^{28}$. Nel caso si trattasse dell'immagine di una divinità, non sarebbe da escludere una divinità ctonia come Persefone, se a Locri Persefone è la divinità protettrice del matrimonio anche in qualità di "Kore/kore"29. A ciò si aggiunga che culti di tipo ctonio ben si addicono ad aree sacre legate alla sfera afrodisia, in associazione con altre figure divine o semi-divine ${ }^{30}$, come dimostra per esempio il caso di Siracusa ${ }^{31}$.

In stridente contrasto con la coroplastica di grandi dimensioni, le piccole terrecotte figurate sono di fattura modesta e sono state prodotte con matrici stanche. Tutto il gruppo di manufatti è inquadrabile entro un arco cronologico piuttosto omogeneo, ossia nel corso del IV sec. a.C. e all'inizio del secolo successivo.

Le iconografie rappresentate sono piuttosto numerose, considerato lo scarsissimo numero di esemplari provenienti dalla stipe. Si può anzi affermare che ogni esemplare è riconducibile ad un tipo diverso. Sono attestate figure femminili, esemplari di c.d. "Temple Boy", protomi, eroti, bambole, votivi anatomici, animali, un'applique.

Le figure femminili sono tutte assise. Se una statuetta di figura seduta con le mani in grembo non consente una lettura sicura per il pessimo stato di conservazione, meglio leggibili sono gli altri esemplari. Due di essi recano tra le mani strumenti musicali (Fig. 28a, b), il tympanon ${ }^{32}$ e il doppio flauto ${ }^{33}$. Inoltre, delle due figure, quella con tympanon è velata (in una foggia che costituisce una variazione della posizione canonica dell'bimation), mentre la suonatrice di doppio flauto - ugualmente seduta - ha i capelli raccolti in un sakkos.

\footnotetext{
${ }^{28}$ Sebbene manchino, in coroplastica, confronti stringenti col nostro esemplare, va ricordato che è su un trono con piedi a forma di zampe leonine che seggono Persefone e Ades e Ades e Dioniso nei pinakes locresi: HigGINs (1967), tav. 38 C-D.

${ }^{29}$ Sourvinou-Inwood (1978), p. 106. Sul mito di Kore quale simbolo dell'iniziazione della donna ved. LinCOLN (1979), p. 223 sq.

30 Per esempio, Kore risulta associata a divinità "generiche" come le Ninfe (Porfirio, Antr. Nymph., 7). Sull'associazione del culto delle Ninfe alle divinità elusine, ved. le interessanti osservazioni di MERTENS HORN (1991), p. 9-28, in part. p. 18-19, che ricorda come, secondo Mnaseas, il culto delle Ninfe fosse sempre associato a Demetra e Kore (Mnaseas, fr. $5=$ Schol. Pyth. IV, 106). In generale - osserva la studiosa (ibidem, p. 18) - proprio l'ampio spettro delle valenze cultuali delle Ninfe, atte a presiedere la fertilità della terra e la fecondità di uomini e animali, fa sì che queste figure fossero generalmente "accostate" ad altre divinità venerate negli stessi luoghi. A conferma di questa usuale associazione, i versi di un poema idillico pastorale, rinvenuto su una lastra marmorea da Akrai: MANGANARO (1981), p. 1079 sq.: [le Ninfe] "abbondano di frutta e con esse ... anche gli altari di altre divinità".

31 Cf. Giudice (1979), p. 343.

32 Numerosi sono i tipi ellenistici di figure femminili con tympanon. L'attributo è talvolta presente, come in questo caso, in esemplari di statuette sedute, attestate per esempio a Timmari: LO PORTO (1991), tav. LII nn. 108-109.

33 Anche in questo caso, ben più comune è l'iconografia che rappresenta figure stanti che suonano l'aulos: cf., a titolo esemplificativo, HigGINs (1954), p. 319, tav. 161 n. 1179.
} 
Fig. 28
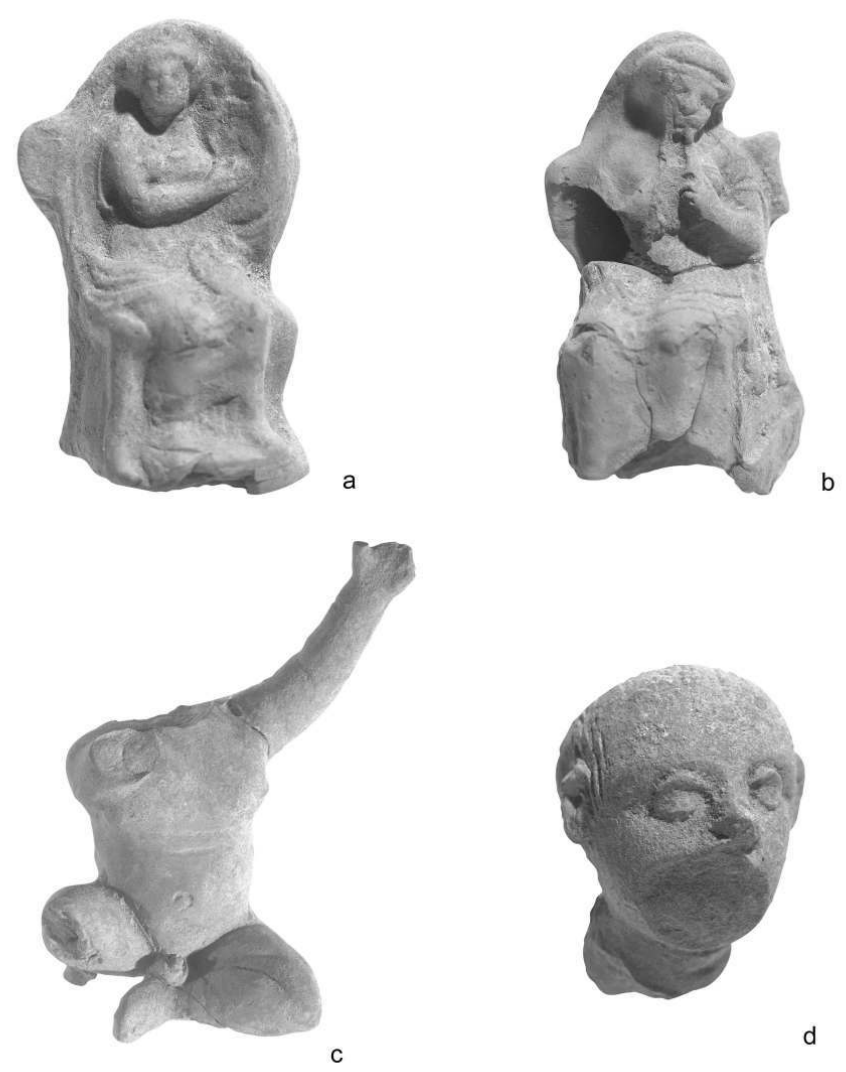

Una figura femminile seminuda (Fig. 29) si distingue dalle altre statuette di piccole dimensioni per la fattura più accurata e raffinata, come si evince dalla resa del panneggio, che scende dalla spalla sinistra per avvolgere i fianchi. Dato lo stato di conservazione del pezzo, la ricostruzione del tipo è difficile, ma la parziale nudità della figura richiama schemi iconografici legati alla figura di Afrodite.

Una statuetta di kourotrophos (Fig. 30), infine, è caratterizzata dalla presenza di un bambino già grande, che poggia la testa sul grembo della madre ${ }^{34}$.

Passando alle altre categorie di terrecotte, due esemplari (Fig. 28c, d) sono riconducibili alla categoria di figurine rodie che rappresentano bambini accoccolati ${ }^{35}$, noti generalmente come "Temple Boys"36.

${ }^{34}$ Cf. un esemplare di Fratte, in cui il bambino stante succhia il latte dal seno della madre: GreCo, Pontrandolfo (1990), p. 112, fig. 201.

35 Higgins (1954), p. 93, tav. 45 (nn. 257, 259).

36 Un inquadramento generale su questa classe di oggetti coroplastici in HADZISTELIOUPRICE (1969), p. 95-111. 


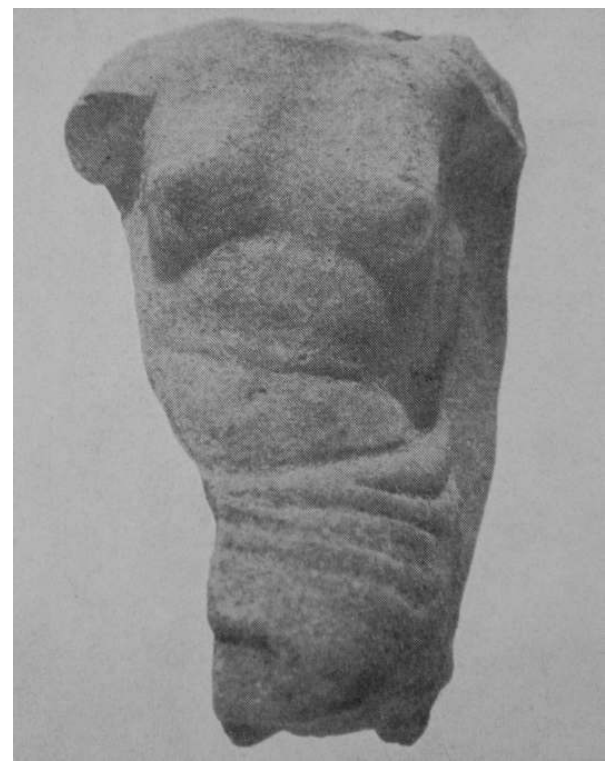

Fig. 29

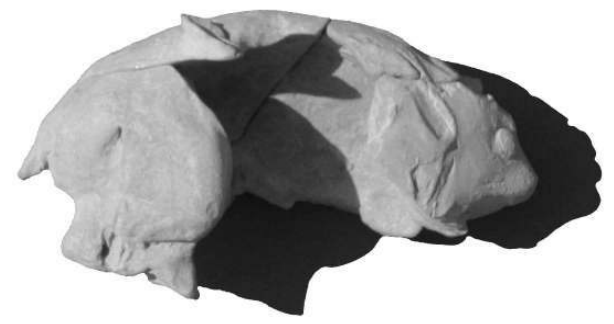

Fig. 31

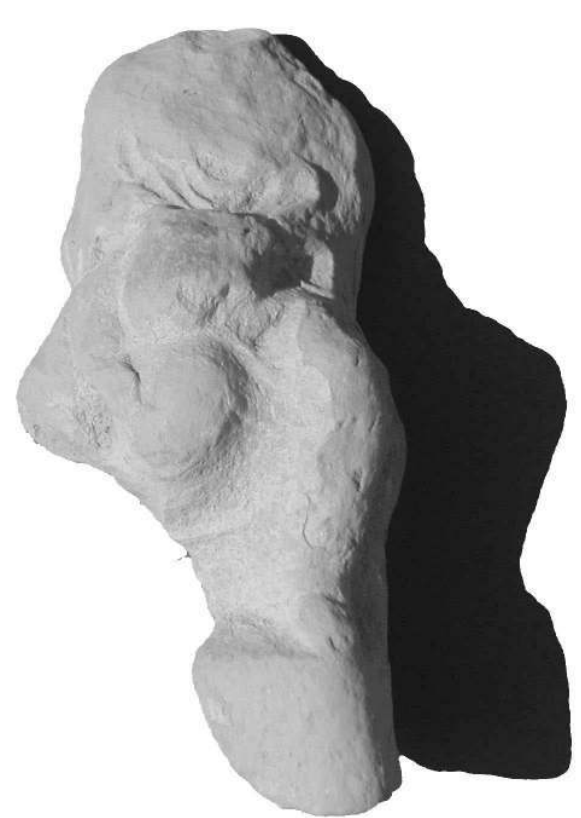

Fig. 30

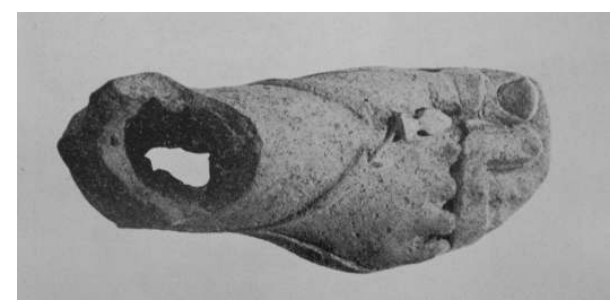

Fig. 32

Un unico esemplare di protome (Fig. 25d) riproduce una figura femminile tagliata all'altezza delle spalle. Il chitone con scollatura a punta e la chioma, resa a sottili ciocche sistemate in due bande divergenti con scriminatura al centro della testa, costituiscono un referente stilistico per datare il pezzo nell'arco del IV sec. a.C.

Molto frammentario è un piccolo erote raffigurato stante, con le gambe incrociate e mollemente appoggiato a un supporto (probabilmente un pilastrino), secondo una comunissima iconografia di età ellenistica, quando cominciano a prediligersi immagini che vedono protagonisti putti ed eroti ${ }^{37}$.

${ }^{37}$ Cf. gli esemplari da Myrina in Burn, Higgins (2001), tav. 53. 
Tra gli animali è attestato un unico esemplare di porcellino (Fig. 31), figura diffusissima nei santuari greci e magno-greci. Sebbene la grossa lacuna sul dorso non consenta una lettura sicura del pezzo, quest'ultimo sembrerebbe recare traccia di una figura (un fanciullo?) che lo cavalcava, secondo un tema squisitamente ellenistico ${ }^{38}$. Infine, a parte un piccolo frammento di braccio identificabile come parte di una bambola per la presenza di un foro all'estremità ${ }^{39}$, si annoverano

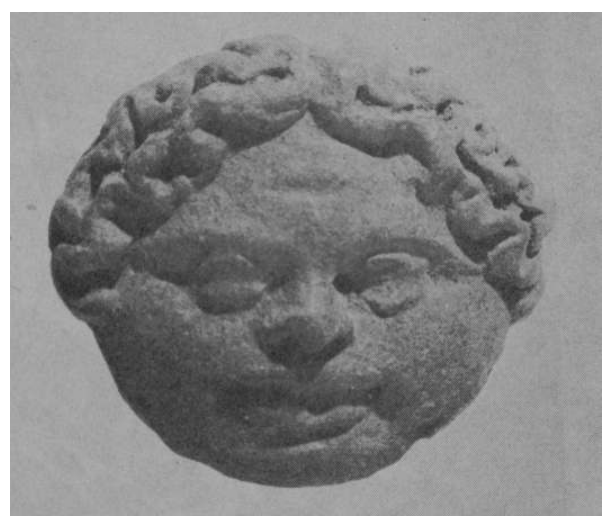

Fig. 33 pochi esemplari di votivi anatomici: dita della mano ed un piede, di fattura, quest'ultimo, particolarmente ricercata soprattutto nella resa del sandalo ${ }^{40}$ e dei particolari anatomici, che rispecchiano una notevole aderenza al vero (Fig. 32).

Un unico esemplare di applique (Fig. 33) presenta strette affinità con gli oscilla gorgonici da Grotta Caruso a Locri ${ }^{41}$. [I.B.]

\section{Divinità e culto nel santuario di Contrada Petrulla. Per una lettura complessiva del dato archeologico}

Prima di passare a considerare nel complesso ruolo e funzione di questo piccolo santuario della chora eracleota e cercare di risalire alla divinità ivi venerata, sono necessarie alcune considerazioni preliminari sui manufatti rinvenuti.

Innanzitutto si vuole porre l'attenzione sullo scarso numero di terrecotte figurate rinvenute, sebbene proprio la coroplastica costituisca generalmente la classe di materiali più rappresentata nei contesti sacri del mondo greco e indigeno dell'Italia meridionale. Ma colpisce ancor più la non ripetitività dei tipi rappresentati: se questo non ci meraviglia per i pochi pezzi pertinenti alla plastica di grandi dimensioni (sia che si voglia ipotizzare una funzione diversa rispetto a quella delle piccole terrecotte figurate, sia che si pensi più semplicemente allo sforzo economico che statue come queste dovevano comportare), è

38 Cf. esemplari di porcellino con putto accovacciato sulla groppa dalla collezione Jatta, databili al IV-III sec. a.C.: VeNTRELLI (2004), p. 85, n. 54; p. 86, n. 55; p. 87, n. 56.

$39 \mathrm{La}$ presenza del foro rende inequivocabile l'attribuzione del pezzo alla categoria delle "bambole", nelle cui articolazioni venivano inseriti fili di ferro che le rendevano "snodabili". Ved. in generale EAA III, s.v. "giocattolo", p. 908-909.

40 Non molto frequente è la resa a rilievo della calzatura nei piedi fittili votivi, come sottolinea FENELLI (1975), p. 227.

${ }^{41}$ Costabile (1991), p. 62, fig. 99. 
invece del tutto inusuale per le statuette di piccolo modulo prodotte a stampo, generalmente abbondanti e rappresentate in innumerevoli varianti nei santuari di tutto il sud della penisola.

Piuttosto che pensare ad una casualità (che difficilmente avrebbe determinato una selezione di "tipi", come sembra essersi verificato), sembra più probabile che tale "selezione" sia stata effettuata in antico. I fittili, infatti, provengono tutti dal deposito ubicato presso l'angolo sud-occidentale del temenos, realizzato verosimilmente, come si è visto, al momento dell'abbandono del santuario. L'eterogeneità tipologica e iconografica del materiale coroplastico sembra quasi potersi ricondurre ad una esplicita volontà di conservare nel tempo, oltre la vita stessa dell'area di culto, le immagini più rappresentative dell'originario repertorio iconografico dell'area sacra, quasi a riassumere simbolicamente tutte le valenze dell'antico culto. Al momento della definitiva chiusura del piccolo santuario rurale si sarebbe proceduto così ad un rituale di abbandono, che deve aver comportato una precisa selezione di manufatti significativi (i più integri ancora esistenti all'interno del recinto?) da deporre all'interno della fossa, destinata ad ospitare piamente i resti delle pratiche liturgiche e votive che si erano svolte nello spazio sacro. Una interpretazione alternativa potrebbe portare invece a vedere nei manufatti rinvenuti, più che il frutto di una selezione operata a posteriori, una originaria diversa funzione degli stessi, ossia non ex-voto offerti in grande quantità e poi - pars pro toto - deposti, bensì oggetti facenti parte dell'arredo sacro del temenos. Oggetti che decoravano il santuario "rappresentativi" del culto ma non necessariamente offerte di fedeli. In ogni caso, nonostante l'unicità dei tipi, deve trattarsi di manufatti altamente significativi al fine dell'identificazione del culto divino.

Se dunque l'eterogeneità tipologica dei pezzi potrebbe trovare una spiegazione nelle dinamiche rituali che devono aver accompagnato l'epilogo della frequentazione religiosa, vale la pena soffermarsi anche sulla differenza qualitativa, più volte sottolineata, degli esemplari rinvenuti, in modo da proporre anche a questo riguardo una ipotesi interpretativa. Come si è detto, se i pezzi pertinenti alla plastica di grande modulo sono certamente il prodotto di ateliers altamente qualificati, le piccole terrecotte figurate rientrano invece nella produzione di terrecotte figurate "a basso costo" appartenenti a tipi noti e ampiamente diffusi in area magnogreca; inoltre, si tratta di figurine prodotte con matrici stanche, che non presentano tracce di lavorazione raffinata. Questa discrepanza qualitativa rimanda senza dubbio ad una significativa differenza "funzionale" dei fittili, semplici ex-voto le piccole terrecotte figurate, statue di culto le figure di dimensioni pari quasi al vero. Le grandi terrecotte sarebbero dunque dei veri e propri agalmata cultuali, che dovevano essere stati esposti in gruppo, all'interno probabilmente di un unico contesto, come si osserverà più avanti. Proprio trattandosi di statue cultuali si potrebbe spiegare l'importazione dei pezzi da un atelier tarantino, mentre le piccole terrecotte riflettono bene la frequentazione decisamente "rurale" del luogo sacro. [I.B.] 
Passando ora a discutere il problema dell'identificazione della figura o delle figure divine che dovevano essere venerate presso il fiume Sinni, è necessario ripercorrere brevemente le precedenti proposte interpretative formulate al riguardo.

Il primo tentativo di identificazione si deve allo scopritore stesso del santuario, Dinu Adamesteanu, il quale ipotizzò che il contesto scavato fosse un heroon, probabilmente quello di Calcante ricordato da Licofrone "presso Siris ed il campo Leuternio... là dove fluiscono le correnti del Siris, che irrigano la piatta campagna Conia" (Alexandra, 968 sq., 982 sq.) ${ }^{42}$. In seguito M. Paola Bini, riconsiderando soprattutto l'iconografia dei vasi a figure rosse, ha avanzato l'ipotesi che la pratica cultuale più che a Calcante fosse da riferire a due figure, forse una maschile e una femminile, le quali sarebbero state titolari ognuna di una delle due strutture scavate all'interno del temenos ${ }^{43}$. Accanto al culto eroico, avrebbe trovato così spazio nel santuario il culto di una divinità ctonia, alla quale rimanderebbero alcuni ex-voto rinvenuti, in particolare la coroplastica di grandi dimensioni. Una nuova ipotesi è stata successivamente avanzata da chi scrive, basandosi solo sul materiale fino ad allora edito: l'area sacra, più che un heroon, sarebbe stata destinata ad un culto femminile di tipo "afrodisio" 44 , rivolto a divinità dalla sfera di competenza molto ampia.

Grazie alla considerazione di tutto il materiale (reso possibile anche dal paziente lavoro di catalogo di una buona parte dei reperti svolto da A. Bruscella per una tesi di laurea, ampliato poi da chi scrive in base alla presa visione di tutto il materiale, soprattutto quello a figure rosse), è stato possibile, di recente e in altra sede, avanzare preliminarmente una nuova proposta, ossia che il santuario sia stato votato al culto delle Ninfe. Figure del panorama religioso greco, queste, ampiamente "versatili" e strettamente legate alla cerchia di varie divinità, tra cui Afrodite e di Dioniso, erano poste di frequente a tutelare la fertilità della natura e degli umani, nonché la fecondità degli animali ${ }^{45}$.

In base alla definitiva valutazione di tutto il materiale presente nella stipe, può essere in questa sede sviluppata l'ipotesi già avanzata, articolando nel complesso l'ipotesi di identificazione.

Un primo dato importante che emerge dalla revisione dei manufatti è la presenza massiccia, tra la ceramica, di forme aperte destinate solitamente al consumo del vino: si tratta essenzialmente di skyphoi e coppe biansate, che insieme ai recipienti atti a contenere e a versare vino e acqua ( 7 crateri, 7 bydriai,

\footnotetext{
42 Adamesteanu (1982), p. 463.

43 BINI (1989), p. 20.

44 OSANNA (1992), p. 100.

45 Sul culto delle Ninfe ved. in generale F. HeichelHEIM, s.v. "Nymphai", RE XVII, 2
} (1937), col. 1527-1599; M. HALm-Tisserant, G. SieBERT, s.v. "Nymphai", LIMC VIII 1 (1997), p. 891-902. Per un quadro esaustivo sul culto delle Ninfe ved. da ultimo LARSON (2001), con bibl. prec. 
3 oinochoai, 2 brocche, un askos), costituiscono il servizio da simposio. Tra le ceramiche individuate sono da citare inoltre le forme da dispensa, documentate $\mathrm{da}$ anfore vinarie, funzionali evidentemente al trasporto del liquido utilizzato nei rituali svolti all'interno dello spazio sacro. Se dunque il richiamo all'uso del vino sembra del tutto evidente, i recipienti esplicitamente connessi con l'acqua, ben attestati nel santuario (bydriai), possono ovviamente costituire sì i contenitori per l'acqua da mescolare al vino, ma nel nostro contesto non possono essere disgiunti dalla significativa presenza di acque "sacre", dall'acqua pura della sorgente (da utilizzare per scopi rituali) a quelle del vicino fiume, cui lo spazio sacro non sembra assolutamente estraneo. Da un lato, dunque, sono da considerare i servizi per il simposio o comunque per il "vino" da utilizzare nella prassi rituale, dall'altro un numero elevato di bydriai da connettere direttamente con l'uso cerimoniale dell'acqua, sul cui significato torneremo più avanti. E che si tratti di vasi rituali, evidentemente per specifici rituali che richiedevano contenitori preziosi, sembra sottolineato dall'inusitata presenza nel nostro contesto proprio di ceramiche decorate a figure rosse, generalmente appannaggio di ricchi corredi funebri più che di aree sacre ${ }^{46}$.

Per quanto riguarda l'uso rituale che si doveva fare di tale sistema vascolare, difficile - se non impossibile - risalire all'effettivo svolgimento delle cerimonie nel nostro contesto. La presenza del servizio da simposio, con tutte le forme necessarie, potrebbe rimandare, comunque, più che a pratiche connesse con il consumo del vino all'interno dello spazio sacro da parte dei fedeli, a pratiche libatorie, che si giovano normalmente proprio dello skyphost7, anche se ovviamente una cosa non esclude l'altra. Va sottolineato al riguardo che una recente ricerca di Sabrina Batino ha messo strettamente in connessione gli skyphoi attici - ampiamente attestati nel nostro contesto - con i rituali (e in primis quelli libatori) relativi a soggetti appartenenti a quella sfera dell'“altro", rispetto al cittadino integrato nella polis, a quel mondo dunque di paides e partheno ${ }^{48}$, scandito dallo svolgimento di rituali iniziatici, che assicurano la crescita normativa dell'individuo, fino al raggiungimento della tappa fondamentale del ciclo esistenziale di ognuno, quello dell'ingresso nella socialità della polis, attraverso il matrimonio per le fanciulle e l'acquisizione dello statuto di cittadino-guerriero per i fanciulli49.

L'esistenza di altre pratiche cerimoniali che prevedevano anche la preparazione di cibi all'interno del santuario è documentata invece dal rinvenimento, tra i manufatti del deposito, di forme specificatamente legate alla "cucina del sacrificio", come quelle in ceramica comune, sia destinate alla mensa e alla

\footnotetext{
46 Una recentissima messa a punto sull'uso di ceramica figurata - nello specifico, attica - nei santuari del mondo greco e italico è ora in FORTUNELLI, MASSERIA (2009).

${ }^{47}$ Burkert (1984), p. 104 sq.; RUdHARDT (1992), p. 240-248.

48 Batino (2002).

${ }^{49}$ BRELICH (1969), passim.
} 
cottura, come le olle (almeno 3 esemplari), i piatti (3 esemplari) e le caccabai (3 esemplari), che alla manipolazione dei prodotti della natura (si pensi al mortaio rinvenuto).

Se dalle forme si passa poi a considerare le immagini, emergono una serie di dati di grande interesse, che sembrano condurre nella medesima direzione indicata dal repertorio delle forme ceramiche. Va sottolineata innanzitutto la significativa presenza di scene riferibili proprio a quel mondo dei fanciulli che già era adombrato dalla ricorrente presenza dello skyphos attico. Particolarmente attestate sono inoltre le immagini che si riferiscono alla sfera dionisiaca; in particolare ricorrono sui vasi satiri e menadi. Accanto a queste vanno menzionate alcune scene dalla spiccata valenza iniziatica e matrimoniale: un frammento di bydria presenta una scena relativa al giudizio di Paride, un cratere il mito di Bellerofonte, un altro probabilmente le nozze di Peleo e Teti.

Il dato che emerge chiaramente dalla considerazione della ceramica è l'insistente riferimento, dunque, alle sfere dionisiaca (alterità rappresentata da Satiri e Menadi) e afrodisia (giudizio di Paride), con un altrettanto evidente riferimento al mondo dei fanciulli e alla loro integrazione nella sfera civica, attraverso la manifestazione del valore (mito di Bellerofonte) e il richiamo al matrimonio (nozze di Peleo e Teti). Sembra evidente che la sfera di azione della divinità e dunque la sua personalità vada ricercata proprio nell'ambito delle figure religiose che presiedono al mondo efebico e alle tappe fondamentali del ciclo esistenziale dei paides e parthenoi, che sfocia nella tappa imprescindibile del matrimonio e la conseguente integrazione nel corpo civico. Ma c'è di più.

Se si considera, al di là della ceramica, il complesso dei manufatti come parte di un sistema coerente, emergono immediatamente altri dati di grande rilevanza per la conoscenza del santuario. Si osservino ad esempio le piccole terrecotte figurate. [M.O.]

Se risulta metodologicamente necessario ribadire la difficoltà di inquadrare un culto attraverso manufatti votivi e/o rituali, tale accorgimento deve valere tanto più nel nostro caso, dove le terrecotte sono particolarmente poco numerose e pertinenti a svariati tipi, che ricorrono una sola volta nel contesto. Quello che si vuole qui sottolineare non è la possibilità di giungere ad una decodificazione certa delle iconografie attestate - considerandole chiavi interpretative per ricostruire la personalità divina e darle un nome - quanto piuttosto la compatibilità dei nostri manufatti coroplastici con quanto viene emergendo dall'analisi delle altre categorie di manufatti. È ovvio, infatti, che l'interpretazione non può scaturire da una sola categoria di oggetti ma dalla considerazione complessiva di tutto il contesto.

Considerando da vicino le poche terrecotte documentate nel nostro santuario, dunque, va fatto innanzitutto riferimento alla kourotrophia, cui allude non solo la 
statuetta di figura femminile con bambino ${ }^{50}$, ma anche la presenza di bambini accovacciati, generalmente collegati all'usanza di utilizzare fanciulli come aiutanti all'interno dei santuari, ma più verosimilmente allusivi di classi di età cui si rivolge in particolare la profilassi della divinità venerata ${ }^{51}$. Al di là di tale evidente valenza curotrofica emerge dalle poche terrecotte la presenza di elementi propri della sfera dionisiaca, cui rimandano le figure di suonatrici di aulos e tympanon ${ }^{52}$, strumenti che richiamano entrambi il $\operatorname{simposio}^{53}$ e di conseguenza il mondo di Dioniso.

Più generico, senza dubbio, il valore semantico attribuibile all'unica protome attestata, che non reca alcun attributo tra le mani. In generale, la protome come rappresentazione abbreviata della figura umana - allude al carattere "ctonio" delle divinità, in quanto simbolo dell'anodos, della risalita dalla terra ${ }^{54}$. Tale tipo di ex-voto si trova pertanto associato a numerose divinità, oltre che alle divinità ctonie per eccellenza, Demetra e Persefone ${ }^{55}$, non risultando certo estranea alla sfera afrodisia. Genericamente alla sfera afrodisia rimandano i soggetti a carattere "erotico" attestati: la figura semipanneggiata, nella quale facilmente potrebbe riconoscersi una raffigurazione di Afrodite, e il piccolo erote con le gambe incrociate. Tali documenti alludono chiaramente alla "sessualità", e quindi all'unione carnale e alla fecondità, rimandando senza dubbio a divinità che simboleggiano e presiedono a quello stadio della vita della donna in cui la sessualità viene per la prima volta riconosciuta a livello sociale, in seguito al passaggio da uno stato verginale in cui era invece non $\operatorname{accettata}^{56}$. Ben si

\footnotetext{
50 Sulla kourotrophia in generale e sulle rappresentazioni di nutrici nel mondo greco, ved. HADZISTELIOU-PRICE (1978).

51 Un'altra spiegazione a questo genere di ex-voto è che si trattasse di doni destinati ai bambini: Schmalz (1974), p. 105 sq.; p. 174 tav. 22 (270); anche se l'assenza degli stessi in sepolture infantili rende poco probante questa lettura.

52 Per quanto consapevoli dell'impossibilità di inquadrare entro schemi interpretativi predefiniti certi dettagli iconografici, sembra suggestivo sottolineare come, delle due statuette di suonatrici, una ha un velo sul capo (che richiama la rappresentazione canonica della nymphe), l'altra ha i capelli trattenuti in un sakkos, secondo la medesima acconciatura che ritroviamo nelle due grandi teste rinvenute.
}

53 LiSSARRAGUE (1987), p. 28.

54 BÉRARD (1974), passim. Bérard ritiene divinità dell'anodos, cioè della "risalita" dalla terra, divinità ctonie quali Kore-Persefone e Dioniso, ma anche Afrodite, alla quale attribuisce un legame con il mondo della fertilità della terra e della fecondità femminile come eredità dell'origine orientale del suo culto; cf. FRIEDRICH (1987), p. 12 sq.

55 ORLANDINI (1963), p. 29-30. Lo studioso sostiene che nella maggioranza dei casi maschere e busti vanno interpretati come raffigurazioni di Demetra e Kore. Ved. inoltre ZuNTZ (1971), p. 152; BELL (1981), p. 85.

56 Secondo lo studio condotto da V. Andò (1996) sulla valenza polisemica del termine nymphe, esso alluderebbe non alla "donna sposata" (per la quale esiste, d'altronde, il termine juví), ma alla "donna che per la sua stessa età (ormai fuori dal tempo prepuberale e nel pieno della sua maturità fisica) vive la sua sessualità" (cit. p. 52), ovvero alla donna, oramai non più parthenos a cui "è socialmente concesso [...] manifestare la propria prossimità alla sfera di Afrodite” (cit. p. 55). 
inserirebbe nel quadro appena proposto, quello di divinità che sovrintendono al passaggio dallo stato verginale a quello di nymphai, la presenza di bambole ${ }^{57}$ (rappresentate da un unico minuto frammento di braccio), da considerare come dono della devota, ancora fanciulla alla vigilia delle nozze ${ }^{58}$. Lo stesso potrebbe dirsi per il porcellino ${ }^{59}$, generalmente dedicato, in sostituzione di veri animali ${ }^{60}$, in rituali legati alla fecondità femminile e in occasione delle nozze ${ }^{61}$, forse proprio per la loro appartenenza ad una specie prolifica ${ }^{62}$.

Un'altra indicazione che restituisce la documentazione coroplastica, ulteriore indizio di una forte valenza "ctonia" del culto, ma soprattutto richiamo esplicito alla sfera della fertilità vegetale, è restituita dai numerosissimi frutti in terracotta rinvenuti nel deposito ${ }^{63}$. Gli ex voto anatomici, infine, alludono alla valenza guaritrice cui la divinità venerata nel nostro santuario non doveva essere certamente estranea. L'aspetto "salutare" del culto è d'altronde anche indiziato dal legame con la fonte, ovvero l'acqua come elemento purificatore, e il vicino fiume. [I.B.]

Se si considera, dunque, il complesso dei manufatti come parte di un sistema coerente, emergono immediatamente altre peculiarità del santuario. Innanzitutto risulta evidente come molta suppellettile, anche nella piccola coroplastica, rimandi a divinità al femminile. Molto dicono le iconografie documentate, dove da un lato si scorgono elementi pertinenti alla profilassi dell'infanzia (la kourotrophos, le terracotte di infanti), dall'altro alle attività connesse alla musica e alla danza (terracotte con timpano e doppio flauto); cui va affiancato l'aspetto di profilassi e della sanatio, indirizzata forse verso una categoria particolare, probabilmente ai fanciulli (ex-voto anatomici). Quello che emerge, nel complesso, è senza dubbio l'estraneità alla sfera demetriaca, come mostra innanzitutto la differenza notevole tra il complesso dei manufatti qui recuperati e quelli dell'altro santuario rurale di S. Maria di Anglona, dove è venerata Demetra ${ }^{64}$. Tale estraneità è ribadita da un lato dall'assenza di riferimenti alla sfera agraria del grano, dall'altro dall'insistenza su aspetti afrodisii e dionisiaci, come documentato anche dall'iconografia vascolare che riproduce il giudizio di Paride, sia dalla presenza impressionante di strumenti legati alla bevanda dionisiaca.

\footnotetext{
57 Sembra suggestiva, a questo proposito, un'ulteriore accezione del termine "nymphe" quale "bambola" (Schol. Teocrito II, 110), come sottolineato da V. Andò (1996), p. 78 nota 123.

58 Costabile (1991), p. 125-126.

${ }^{59}$ Più numerose sono tuttavia le dediche di porcellini e cinghiali in santuari di tipo demetriaco.

${ }^{60}$ Sulla consuetudine di consacrare animali in terracotta, ved. in generale PESETTI (1994), p. 31 sq.

61 Varrone, De re rustica II, 2, 4, 9. Per attestazioni di porcellini dedicati a divinità quali Afrodite e Dioniso, ved. Blinkenberg (1931), col. 583; Costabile (1991), p. 149.

${ }^{62}$ Della Torre, Ciaghi (1980), p. 66 n. 24.

63 OSANNA (1992), p. 100.

64 OsanNA et al. (2008), p. 63-64.
} 
Se questo è il panorama che si profila dall'analisi dei manufatti ceramici e della piccola coroplastica, osservazioni importanti ai fini dell'individuazione del culto consentono le tre statue di figure femminili recuperate. Se è impossibile avanzare ipotesi sulla ricostruzione del tipo cui ricondurre la testa con diadema, vale la pena soffermarsi sulle due teste iconograficamente simili, caratterizzate da acconciatura con sakkos e corona di ghiande, da ricostruire come figure femminili panneggiate sedute e riccamente abbigliate. La rappresentazione di una giovane donna vestita in maniera ricercata, con un copricapo tipico delle donne "comuni", potrebbe essere, come accennato, una rappresentazione volutamente generica della nymphe, la giovane alla vigilia delle nozze, cui potrebbe del resto alludere proprio la presenza della stephane. Questo elemento rimanda ad un aspetto iniziatico, che doveva essere di primaria importanza, come già sottolineato, nell'ambito del contesto sacro che si sta analizzando, dove una delle caratteristiche principali del culto e dunque della divinità qui venerata era quella di accompagnare le fanciulle al passaggio delle nozze.

Come si è visto, le tre teste sono state interpretate come statue di culto (come dimensioni e qualità indicherebbero). Nel caso l'interpretazione delle tre grandi teste abbia colto nel segno, avremmo già una concreta indicazione riguardo il culto qui ospitato: si tratterebbe infatti della venerazione di un gruppo divino, di divinità al plurale e non di una singola personalità divina, come potrebbe essere ad esempio Afrodite. Le tre statue, che potevano essere state ospitate nella edicola ricostruita nello spazio antistante l'altare, significativamente rivolte verso il fulcro del santuario, richiamerebbero così divinità venerate in una triade, e immaginate come fanciulle colte nel momento significativo della transizione matrimoniale, come l'elegante abbigliamento e la preziosa acconciatura con stephane potrebbe indicare ${ }^{65}$.

La presenza di tre statue femminili, verosimilmente tutte assise in trono, rappresentate come fanciulle, risulta ben compatibile con un culto delle Ninfe, come avanzato in altra sede: sono proprio le Ninfe ad essere, per definizione, venerate al plurale, e solitamente in una triade. L'iconografia del resto di fanciulla /nymphe, come sopra avanzato, potrebbe già spingere verso la identificazione con le nymphai per antonomasia, ossia proprio le Ninfe, in un sottile gioco di allusioni che permette alla fedele nell'atto del passaggio esistenziale fondamentale di identificarsi con la divinità protettrice del suo passaggio di status. A sostegno di tale ipotesi va richiamata tutta una serie di elementi che nel nostro contesto risultano assolutamente compatibili con un culto delle Ninfe. Se si considera al riguardo la ampia raccolta di dati offerta dal recente lavoro di Jennifer Larson, in particolare gli aspetti che secondo la studiosa sono tipici delle Ninfe e permettono di riconoscerle, si noterà quanto questi elementi ricorrano tutti all'interno del nostro contesto ${ }^{66}$.

\footnotetext{
$65 \mathrm{Al}$ riguardo vedi le importanti osservazioni di GRAEPLER (1994), p. 284 sq.

${ }^{66}$ LARSON (2001), p. 3-8.
} 
Innanzitutto si può prendere in considerazione l'ubicazione dell'area sacra, il suo rapporto con il paesaggio agrario della chora eracleota, ma in particolare con quegli spazi di "passaggio", dove l'elemento pastorale ha un'importanza decisiva. Inoltre, significativamente, il rapporto stretto con aspetti fortemente caratterizzanti l'ambiente, come sorgente e fiume. Del tutto scontato al riguardo è il richiamo alla predilezione delle Ninfe per contesti rurali, ameni, dove grotte e acque definiscono il paesaggio circostante. L'esistenza di una sorgente e la vicinanza alla sponda del Sinni rimandano ad una delle caratteristiche principali delle Ninfe, il loro legame, o meglio l'identificazione vera e propria con le sorgenti da un lato e la particolare associazione con $i$ fiumi. Le Ninfe, infatti, non solo sovrintendono ma si identificano con l'elemento liquido, promettendo la fecondità umana e naturale proprio attraverso il potere vitale insito nell'acqua ${ }^{67}$; spesso ritornano nel mito come figlie di fiumi (si pensi tra gli altri all'Acheloo, dio-fiume per eccellenza, o all'Asopo beotico), mentre l'associazione stretta con le fonti, più volte richiamata dal mito, è evidente nella stessa onomastica che di frequente propone nomi con il suffisso -rhoe, allusivo delle acque correnti ${ }^{6}$.

L'insistenza con cui ritorna nel complesso dei materiali l'allusione alla sfera pre-nuziale - e in particolare l'aspetto curotrofico - e al matrimonio, come tappa fondamentale del ciclo esistenziale che corona una crescita normativamente scandita degli umani, attraverso passaggi esistenziali importanti, è un altro tratto che suggerisce una decisa compatibilità del nostro luogo sacro con il culto generalmente prestato alle Ninfe. Non è certo un caso che nel mito è proprio alle Ninfe che sia demandata la profilassi degli infanti, i quali vengono accompagnati nelle prime fasi dell'esistenza, per poi essere seguiti nelle varie fasi del ciclo esistenziale ${ }^{69}$. Sono, le Ninfe, divinità kourotrophoi per eccellenza ${ }^{70}$, le quali presiedono alla nascita e al primo sviluppo degli infanti, come ricorda già Esiodo (Teogonia, 346-348); sono le Ninfe, del resto, che lavano Hermes appena nato (Pausania, VIII, 61, 1) o che accudiscono Zeus e Dioniso infanti (Pausania, IV, $33,1)$. Non meraviglia dunque ritrovare immagini relative a fanciulli qui ad Heraklea come in altri santuari delle Ninfe, a cominciare da quello dall'antro coricio a Delfi. Ampiamente nota è del resto la sfera di azione di tali divinità nell'ambito dei riti di passaggio tanto dei fanciulli quanto delle donne nel momento fondamentale del loro ciclo esistenziale, le nozze ${ }^{71}$. Lo stretto legame tra sposa e divinità è evocato dallo stesso nome che designa entrambe: nymphe

\footnotetext{
${ }^{67}$ La connessione delle Ninfe con le fonti è già nota ad Omero, che ricorda un altare delle Ninfe presso una fonte di acqua fresca $(\mathrm{O} d$. XIV, 435). Nell'immaginario mitico greco, inoltre, queste figure sono dispensatrici di acqua (Pindaro, Ol. 12, 87). Sul carattere "acquatico" delle Ninfe ved. in generale LARSON (2001), p. 8-9, con bibliografia precedente.

${ }^{68}$ Documentazione in LARSON (2001), p. 4, 98-100.

${ }^{69}$ LARSON (2001), p. 43-44, 111.

${ }^{70} \mathrm{Si}$ veda per es. Eschilo, Xantriai, TrGF 4 F 168. 16-25; Euripide, Elettra, 625-626; Esiodo, Teogonia, 346-366.

${ }^{71} \mathrm{Si}$ veda per es. Plutarco, Mor., 772b.
} 
(Iliade XVIII, 492; Odissea XI, 447). Si pensi alla eccezionale documentazione ateniese proveniente dal santuario della Ninfa, presso le pendici meridionali dell'acropoli, destinato per l'appunto allo svolgimento da parte delle donne di riti prenuziali $^{72}$.

Altro aspetto che emerge evidente, tanto dalle terrecotte quanto dall'iconografia vascolare della ceramica a figure rosse, è il riferimento puntuale e pervasivo alla sfera dionisiaca. In particolare satiri e menadi dominano le immagini della ceramica, in particolare degli skyphoi. Anche in questo caso è facile il richiamo al mondo "promiscuo" delle Ninfe, dove il rapporto con i Satiri è parte integrante del loro rapporto con l" "altro", con il mondo pastorale e soprattutto, ancora una volta, con la loro valenza iniziatica. Si potrebbe continuare nella puntuale disamina dei dati e delle caratteristiche della nostra area sacra che potrebbero richiamare il mondo delle Ninfe, ma mi sembra che già gli elementi finora richiamati siano sufficienti a restituire una identità divina alle figure per le quali era stato eretto il piccolo temenos nella valle del Sinni. Un culto destinato ad una vita breve, travolto probabilmente dalle trasformazioni di quel territorio cui le Tavole di Heraklea fanno ampio cenno. [M.O.]

University of Alberta

Ilaria BATTILORO

Department of History and Classics

2-28 Henry Marshall Tory Building

Edmonton, Alberta

CANADA T6G 2H4

E-mail:ilaria@ualberta.ca

Antonio BRUSCELLA

Università degli Studi della Basilicata

Scuola di Specializzazione in Archeologia di Matera

Via San Rocco 1

I - 75100 MATERA

E-mail: antoniobruscella@hotmail.it

Università degli Studi della Basilicata

Massimo OSANNA

Scuola di Specializzazione in Archeologia di Matera

Via San Rocco 1

I - 75100 MATERA

E-mail:massimo.osanna@unibas.it

${ }^{72}$ Travlos (1972), p. 361-364. 


\section{Elenco del materiale illustrativo}

Fig. 1 Heraklea e la sua chora

Fig. 2 Planimetria del complesso sacro

Fig. 3 Altare

Fig. 4 Altare: ipotesi ricostruttiva

Fig. 5 Elementi architettonici appartenenti al naiskos, assemblati al Museo Archeologico Nazionale della Siritide di Policoro

Fig. 6 Planimetria delle strutture all'interno del temenos

Fig. 7 Skyphos a vernice nera

Fig. 8 Coppa a vernice nera

Fig. 9 Coppetta a vernice nera

Fig. 10 Coppetta a vernice nera

Fig. 11 Kylix

Fig. 12 Hydria a figure rosse

Fig. 13 Frammento di cratere a figure rosse

Fig. 14 Frammento di cratere a figure rosse

Fig. 15 Frammento di skyphos a figure rosse

Fig. 16a Skyphos a figure rosse, lato A

Fig. 16b Skyphos a figure rosse, lato B

Fig. 17 Frammento di vaso a figure rosse

Fig. 18 Frammento di vaso a figure rosse

Fig. 19 Frammento di lekythos a figure rosse

Fig. 20 Frammenti di vasi a figure rosse

Fig. 21 Frammento di cratere a figure rosse con raffigurazione del mito di Bellerofonte e la Chimera

Fig. 22 Frammento di bydria a figure rosse con raffigurazione del Giudizio di Paride

Fig. 23 Frammento di cratere a figure rosse con raffigurazione di un corteo nuziale

Fig. 24 Frammenti di skyphoi a figure rosse

Fig. 25 a. Testa femminile con sakkos; b. testa femminile con diadema; c. testa femminile con sak.kos; d. protome femminile

Fig. 26 Braccio di figura femminile seduta

Fig. 27 Parte del trono di figura femminile seduta

Fig. 28 a. Statuetta femminile con tympanon; b. statuetta femminile con aulos; c. d. esemplari di "Temple Boy"

Fig. 29 Statuetta femminile semipanneggiata

Fig. 30 Statuetta di kourotrophos

Fig. 31 Porcellino fittile

Fig. 32 Ex-voto anatomico

Fig. 33 Applique fittile 


\section{Bibliografia}

Adamesteanu D. (1977), "L'antico aspetto del terreno delle colonie di Metaponto, Heraclea, Sybaris e di altri centri indigeni della Lucania", in P.-M. Duval, ED. Frezouls (a cura di), Thèmes de recherches sur les villes antiques de l'Occident (Strasbourg, $1^{\mathrm{er}}-4$ octobre 1971), Paris (Coll. intern. du CNRS, 542), p. 347-377.

Adamesteanu, D. (1982), "Un Heroon sulla valle del Sinni?”, in APARCHAI. Nuove Ricerche e Studi sulla Magna Grecia e la Sicilia antica in onore di Paolo Enrico Arias, Pisa, p. 459-464.

Amandry, P. (1984), Le culte des Nymphes et de Pan à l'antre corycien, Paris (BCH, suppl. 9, 2), p. 395425.

ANDÒ, V. (1996), "Nymphe. La sposa e le Ninfe”, QUCC 52, I, p. 47-79.

BATINO, S. (2002), Lo skyphos attico dall'iconografia alla funzione, Napoli.

BeLL, M. (1981), Morgantina Studies I, The Terracottas, Princeton.

Belli Pasqua, R. (1995), Taranto. La scultura in marmo e in pietra, IV, 1 (Catalogo del Museo Archeologico Nazionale di Taranto), Taranto.

BÉRARD, C. (1974), Anodoi. Essai sur l'imagerie des passages chthoniens, Neuchatel.

Bernabò BREA, L. (1952), "I rilievi tarantini in pietra tenera", RLA 1, p. 5-241.

BINI, M.P. (1989), "Il territorio di Eraclea nel IV-III sec. a.C.", in Studi su Siris-Eraclea, Roma (ArchPer 9), p. 15-21.

BlinkenberG, Chr. (1931), Lindos. Fouilles et découvertes 1902-1914, I, Les petits objets, Berlin/ Copenhagen.

Bouma, J. (1996), Religio votiva. The Archaeology of Latial Votive Religion, I, Groningen.

Breglia Pulci Doria, L. (2002), "Elmo frigio, Atena, Ilias, Palladio", in L. Cerchiai (a cura di), L'iconografia di Atena con elmo frigio in Italia meridionale (Atti della Giornata di Studi, Fisciano 1998), Napoli, p. 104-105.

Brelich, A. (1969), Paides e Parthenoi, Roma.

Burkert, W. (1984), Anthropologie des religiösen Opfers: die Sakralisierung der Gewalt, München.

Burn, L., Higgins, R. (2001), Catalogue of Greek Terracottas in the British Museum, III, London.

Cambitoglou, A., Chamay, J., (1997), Céramique de Grande Grèce. La Collection de fragments Herbert A. Cabn, Zürich.

CARTER, J.C. (1987), "Agricoltura e pastorizia, in Magna Grecia tra Bradano e Basento", in G. Pugliese Carratelli (a cura di), Magna Grecia. Lo sviluppo politico, sociale ed economico, Milano, p. 173-212.

CostabiLe, F. (1991), I ninfei di Locri Epizefiri. Architettura, culti erotici, sacralità delle acque, Soveria Mannelli.

DE JuLiIS, E.M. (2004), "La ceramica a figure rosse magnogreca. Origine della ceramica italiota a figure rosse e sua diffusione in Puglia", in G. Sena Chiesa, E.A. Arslan (a cura di), Miti greci. Archeologia e pittura dalla Magna Grecia al Collezionismo, Milano, p. 145-149.

Della Torre, O., Ciaghi, S. (1980), Terrecotte figurate ed architettoniche del Museo Nazionale di Napoli, Napoli.

DinsmoOR, W.B. (1950), The Architecture of Ancient Greece, London.

Etienne, R., Dinhaet, M.T. (1991), L'Espace sacrificiel dans les civilisations méditerranéennes de l'antiquité (Atti del Colloquio, Lione 1988), Lione.

Feneldi, M. (1975), "Contributo per lo studio del votivo anatomico. I votivi anatomici di Lavinio", ArchClass 27, 2, p. 206-252.

Forti, L., Stazio, A. (1983), "Vita quotidiana dei Greci d'Italia", in G. Pugliese Carateldi (a cura di), Megale Hellas, Milano, p. 641-713. 
Fortunelli, S., MASSERIA, C. (a cura di) (2009), Ceramica attiva dai santuari della Grecia, della Ionia e dell'Italia (Atti del Convegno di Perugia, 14-17 marzo 2007), Venosa.

Friedrich, P. (1987), The Meaning of Aphrodite, Chicago.

Giorgi, M. et al. (1988), Forentum I. La necropoli di Lavello, Venosa.

Giudice, F. (1979), "La stipe di Persefone a Camarina", Mon AL 49, Serie Misc. II, p. 277-354.

GraePler, D. (1994), Tonfiguren in Grab, München.

Greco, G., Pontrandolfo, A. (1990), Fratte. Un insediamento etrusco-campano, Modena.

Hadzisteliou-Price, T. (1969), "The crouching child and 'Temple Boys", ABS A 64, p. 95-111.

Hadzisteliou-Price, T. (1978), Kourotrophos. Cult and representations of the Greek nursing deities, Leiden.

Higgins, R.A. (1954), Catalogue of the Terracottas in the Department of Greek and Roman antiquities, British Museum, I, London.

Higgins, R.A. (1967), Greek Terracottas, London.

IACOBOnE, C. (1988), Le stipi votivi di Taranto (scavi 1885-1934), (Corpus delle stipi votive in Italia, 2. Regio II, 1, Archaeologica 2), Roma.

ISLER KERÉNYI, C. (2004), "Religione e misteri. Dioniso ed Eros nella ceramica apula", in G. Sena Chiesa, E.A. Arslan (a cura di), Miti greci. Archeologia e pittura dalla Magna Grecia al collezionismo, Milano, p. 246-248.

Jeanmaire, H. (1949), "Le Satyre et la Ménade. Remarques sur quelques textes relatifs aux danses « orgiaques »", RA 29-30, p. 463-473.

Johnson, F.P. (1955), “A note on Owl-skyphoथ”, AJA 59, p. 119-124.

Josep, R. (1997), Las casas del Alma. Maquetas architectónicas de la Antigüedad (5500 a.C.-300 d.C.), Barcelona.

KERÉNYI, K. (1984), Gli dei e gli eroi della Grecia, [trad. it.] Milano.

LARSON, J. (2001), Greek nymphs: myth, cult, lore, Oxford-New York.

Larson, J. (2007), "A Land Full of Gods: Nature Deities in Greek Religion”, in D. Ogden (a cura di), A Companion to Greek Religion, Malden, p. 56-70.

LA Torre, G.F. (2002), Un tempio arcaico nel territorio dell'antica Temesa. L'edificio sacro in località Imbelli di Campora San Giovanni, (Corpus delle stipi votive in Italia, Regio III, 4), Roma.

LatTanzi, E. et al. (1996), Santuari della Magna Grecia in Calabria, (Catalogo delle Mostre), Napoli.

LeTTA, C. (1971), Piccola coroplastica metapontina nel Museo Archeologico di Potenza, Napoli.

LinCOLN, B. (1979), "The rape of Persephone: a Greek scenario of women's initiation”, HThR 72, p. 223-235.

Lippolis, E. (1987), “Organizzazione delle necropoli e struttura sociale nell'Apulia ellenistica. Due esempi: Taranto e Canosa", in H. von Hesberg, P. Zanker (a cura di), "Römische Gräberstrassen" (Selbstdarstellung, Status, Standard. Kolloquium in München vom 28. bis 30. Oktober 1985, quad. 96), München, p. 139-154.

Lippolis, E. (1994), "La tipologia dei semata", in A. Dell’Aglio, E. Lippolis (a cura di), Catalogo del Museo Nazionale Archeologico di Taranto, III, 1. Taranto. La necropoli: aspetti e problemi della documentazione archeologica tra VII e I sec. a.C., Taranto, p. 109-128.

Lippolis, E. (1996), "Lo stile proto-apulo e apulo antico e medio", in E. Lippolis (a cura di), I Greci in Occidente: Arte e artigianato in Magna Grecia, Napoli, p. 377-393.

Lissarague, F. (1987), Un flot d'images. Une esthétique du banquet grec, Paris [trad. it. Bari 1989].

Lo PORTO, F.G. (1991), Timmari: l'abitato, le necropoli, la stipe votiva, (Archaeologica 98), Roma.

Manganaro, A. (1981), "L'oracolo di Maie per una carestia in territorio siracusano", ASNP, 11,4 , ser. 3, p. 1069-1082. 
Mertens Horn, M. (1991), "Una nuova antefissa a testa femminile da Akrai ed alcune considerazioni sulle Ninfe in Sicilia", BA 76, ser. VI, p. 9-28.

Masseria, C. (1999), ““Et Venerem et proelia destinat” (Hor. carm. III, 13, 5). Riti di passaggio in un santuario di Banzi", Ostraka VIII, 2, p. 469-490.

Moret, J.M. (1978), "Le jugement de Pâris en Grande-Grèce : mythe et actualité politique”, $A K$ 21, p. 76-98.

Mugione, E. (2000), Miti della ceramica attica in Occidente. Problemi di trasmissioni iconografiche nelle produzioni italiote, Taranto.

Mugione, R. (2002), "Le immagini di Atena con elmo frigio nella ceramica italiota", in L. Cerchiai (a cura di), L'iconografia di Atena con elmo frigio in Italia meridionale (Atti della Giornata di Studi), Fisciano 12 giugno 1998, Napoli, p. 63-80.

Museo (Il) di Taranto. 1988. Cento anni di archeologia, Martina Franca.

Nilsson, M.P. (1952), A History of Greek Religion, Oxford.

Orlandini, P. (1963), “Gela. La stipe votiva arcaica del Predio Sola”, MonAL 46, p. 1-78.

Orlandini, P. (1983), "Le arti figurative", in G. Pugliese Caratelli (a cura di), Megale Hellas, Milano.

Osanna, M. (1992), Chorai coloniali da Taranto a Locri. Documentazione archeologica e ricostruzione storica, Roma.

Osanna, M., NAva, M.L. (2001), Rituali per una Dea Lucana. Il santuario di Torre di Satriano, Afragola.

Osanna, M. (2001), “Azioni rituali e offerte votive”, in Osanna, NAVA (2001), p. 113.

Osanna, M. et alii (2008), Culti greci in Occidente, II. Eraclea, Taranto.

PesetTI, S. (1994), Capua preromana. Terrecotte votive VI: Animali, frutti, giocattoli, pesi da telaio, Firenze.

PIANU, G. (1996), "L’agorà di Eraclea Lucana”, in B. OTTO (a cura di), Herakleia in Lukanien und das Quellheiligtum der Demeter, Innsbruck, p. 71-82.

Pirenne-Delforge, V. (1994), L'Aphrodite grecque. Contribution à l'étude de ses cultes et de sa personnalité dans le panthéon archaïque et classique, Athènes/Liège (Kernos, suppl. 4).

Pontrandolfo, A. et al. (1988), "Semata e naiskoi nella ceramica italiota", AION (acheol.) 10, p. 181-201.

POuZADOux, C. (2002), Tra mondo greco e mondo lucano. Immagine e mito nella ceramografia del IV sec. a.C., in M. Osanna, M.L. NAVA (a cura di), Immagine e mito nella Basilicata antica (Catalogo della Mostra, Potenza 2002), Venosa, p. 121-128.

QuilicI, L. (1967), Siris-Heraclea, Forma Italiae, Regio III, vol. I, Roma.

RESTANI, D. (1991), "Dionysos tra aulos e kitara: un percorso di iconografia musicale", in F. Berti, Dionysos. Mito e mistero (Atti del Convegno, Comacchio 1989), Ferrara, p. 379-395.

RudHARDT, J. (1992), Notions fondamentales de la pensée religieuse et actes constitutifs du culte dans la Grèce classique, Paris.

Russo Tagliente, A. (2000), Armento. Archeologia di un centro indigeno (BA XXXV-XXXVI), Roma.

Schmalz, B. (1974), Terrakotten aus dem Kabirenheiligtum bei Theben, Bd. 5: Menschenähnliche Figuren und Gerät, Berlin.

Sourvinou-Inwood, C. (1978), "Persephone and Aphrodite at Locri: a model for personality definitions in Greek religion”, JHS 98, p. 101-121.

Sparkes, B.A., TalcotT, L. (1970), The Athenian Agorà, XII, Black and Plain Pottery of $6^{\text {th }}, 5^{\text {th }}$ and $4^{\text {th }}$ Centuries B.C., Princeton.

STACCIOLI, R.A. (1968), Modelli di edifici etrusco-italici. I modelli votivi, Firenze. 
STUCCHI, S. (1975), Architettura cirenaica, (Monografie di archeologia libica 9), Roma.

Travlos, J. (1972), Pictorial Lexicon of Ancient Athens, New York.

Trendall, A.D. (1953), Vasi antichi dipinti del Vaticano. Vasi italioti ed etruschi a figure rosse, Fasc. 1, Città del Vaticano.

Ventrelli, D. (2004), Le terrecotte figurate del Museo Nazionale Jatta di Ruvo, Bari.

WEBER, M. (1990), Baldachine und Statuenschreine, Rom.

ZunTZ, G. (1971), Persephone, Oxford. 